\title{
HOPuppis: Not a Be Star, but a Newly Confirmed IW And-type Star
}

\author{
Chien-De Lee ${ }^{1}\left(\mathbb{D}\right.$, Jia-Yu Ou $^{1}$, Po-Chieh $\mathrm{Yu}^{1,2}{ }^{10}$, Chow-Choong Ngeow ${ }^{1}\left(\mathbb{0}\right.$, Po-Chieh Huang ${ }^{1}$, Wing-Huen $\mathrm{Ip}^{1}$, \\ Franz-Josef Hambsch ${ }^{3,4,5}$, Hyun-il Sung ${ }^{6}$ (10), Jan van Roestel ${ }^{7}$ (1) Richard Dekany ${ }^{8}$, Andrew J. Drake ${ }^{7}$, Matthew J. Graham ${ }^{7}$ (1),

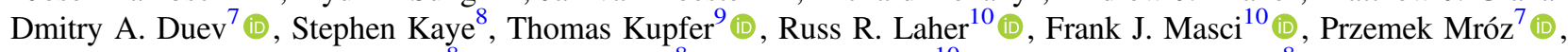 \\ James D. Neill ${ }^{8}$ (1), Reed Riddle ${ }^{8}$ (i), Ben Rusholme $^{10}{ }^{(1)}$, and Richard Walters ${ }^{8}$ (1) \\ ${ }^{1}$ Graduate Institute of Astronomy, National Central University, Jhongli 32001, Taiwan; cngeow@astro.ncu.edu.tw \\ ${ }^{2}$ College of General Studies, Yuan-Ze University, Chung-Li 32003, Taiwan \\ ${ }^{3}$ American Association of Variable Star Observers (AAVSO), Cambridge, MA, USA \\ ${ }^{4}$ Vereniging Voor Sterrenkunde (VVS), Oostmeers 122 C, B-8000 Brugge, Belgium \\ ${ }^{5}$ Bundesdeutsche Arbeitsgemeinschaft für Veränderliche Sterne e.V. (BAV), Munsterdamm 90, D-12169 Berlin, Germany \\ ${ }^{6}$ Korea Astronomy and Space Science Institute (KASI), Bohyunsan Optical Astronomy Observatory (BOAO), Youngcheon, Gyungbuk 38812, Republic of Korea \\ Division of Physics, Mathematics, and Astronomy, California Institute of Technology, Pasadena, CA 91125, USA \\ ${ }^{8}$ Caltech Optical Observatories, California Institute of Technology, Pasadena, CA 91125, USA \\ ${ }^{9}$ Kavli Institute for Theoretical Physics, University of California, Santa Barbara, CA 93106, USA \\ ${ }^{10}$ IPAC, California Institute of Technology, Pasadena, CA 91125, USA \\ Received 2020 August 6; revised 2021 February 17; accepted 2021 February 18; published 2021 April 15
}

\begin{abstract}
HO Puppis (HO Pup) was considered as a Be-star candidate based on its $\gamma$ Cassiopeiae-type light curve, but lacked spectroscopic confirmation. Using distance measured from Gaia Data Release 2 and the spectral-energydistribution fit on broadband photometry, the Be-star nature of HO Pup is ruled out. Furthermore, based on the 28,700 photometric data points collected from various time-domain surveys and dedicated intensive-monitoring observations, the light curves of HO Pup closely resemble those of IW And-type stars (as pointed out by Kimura et al.), exhibiting characteristics such as a quasi-standstill phase, brightening, and dips. The light curve of HO Pup displays various variability timescales, including brightening cycles ranging from 23 to 61 days, variations with periods between 3.9 days and 50 minutes during the quasi-standstill phase, and a semiregular $\sim 14$ day period for the dip events. We have also collected time-series spectra (with various spectral resolutions), in which Balmer emission lines and other spectral lines expected for an IW And-type star were detected (even though some of these lines were also expected to be present for Be stars). We detect Bowen fluorescence near the brightening phase, and that can be used to discriminate between IW And-type stars and Be stars. Finally, despite only observing for four nights, the polarization variation was detected, indicating that HO Pup has significant intrinsic polarization.
\end{abstract}

Unified Astronomy Thesaurus concepts: Dwarf novae (418); Be stars (142); Sky surveys (1464); Time series analysis (1916)

Supporting material: machine-readable table

\section{Introduction}

Be phenomena are the photometric and spectroscopic variability seen in the main-sequence luminous rapid rotators, known as Be stars, with a luminosity class III-V. In recent years, we have studied the evolutionary effect on the formation of Be stars in open clusters (Yu et al. 2015, 2016, 2018) using the Palomar Transient Factory (Law et al. 2009) and the intermediate Palomar Transient Factory (iPTF; Kulkarni 2013). The Zwicky Transient Facility (ZTF; Bellm et al. 2019; Graham et al. 2019; Masci et al. 2019) came after iPTF, and its improved data can extend our investigation on the variability of Be stars (Ngeow et al. 2019), especially for the Be stars and Be-star candidates at the faint end $(m>13 \mathrm{mag})$, which were largely excluded from previous works (e.g., in Labadie-Bartz et al. 2017). Together with accompanying time-series spectroscopic data, we have a new opportunity to explore the fundamental time-domain nature of Be stars.

Here we report the photometric characteristics of HO Puppis (HO Pup, $\alpha_{\mathrm{J} 2000}=7^{\mathrm{h}} 33^{\mathrm{m}} 54^{\mathrm{s}} .18, \delta_{\mathrm{J} 2000}=-15^{\circ} 45^{\prime} 38^{\prime \prime}$ '28) as a result of our investigations of the variability of Be stars with ZTF. HO Pup is listed as a Be star in the SIMBAD database (Manek 1997) and hence is included in our list of Be-star candidates, in which the classification is based on its $\gamma$
Cassiopeiae (GCAS) type variability recorded by Samus et al. (2017) - a class of variable stars that exhibit eruptive irregular variability that is not easily classified further. Some early literature even suggested that HO Pup was a possible type Ia supernova with $V$-band photometry varying between $12.7 \mathrm{mag}$ and 14.2 mag (Kukarkin et al. 1971). As presented in Figure 1, two highly unusual $\sim 2.5$ mag dips of HO Pup were observed by ZTF in 2017 November. In the $r$ band, the ZTF data cover the full brightness minimum of the two dips at 16 mag from near the minimum to maximum brightness within two days because of the high-cadence sampling. These two events were also witnessed by the All-Sky Automated Survey for Supernovae (ASAS-SN, Shappee et al. 2014; Kochanek et al. 2017). In addition to these two events recorded by ZTF and ASAS$\mathrm{SN}$, we have found more dips based on the literature and archival data since 2009 (see Section 2). It is very unusual to observe these dips with amplitudes of $\sim 2.5$ mag in typical Be stars, and hence HO Pup caught our attention and merits further investigation.

The variability behavior of HO Pup was also considered as a special type of cataclysmic variable (CV). The ASAS-SN light curve of HO Pup was discussed in a recent work by Kimura et al. (2020a). In their study, HO Pup was classified as a dwarf nova (DN) with a unique heartbeat-like oscillation in its 

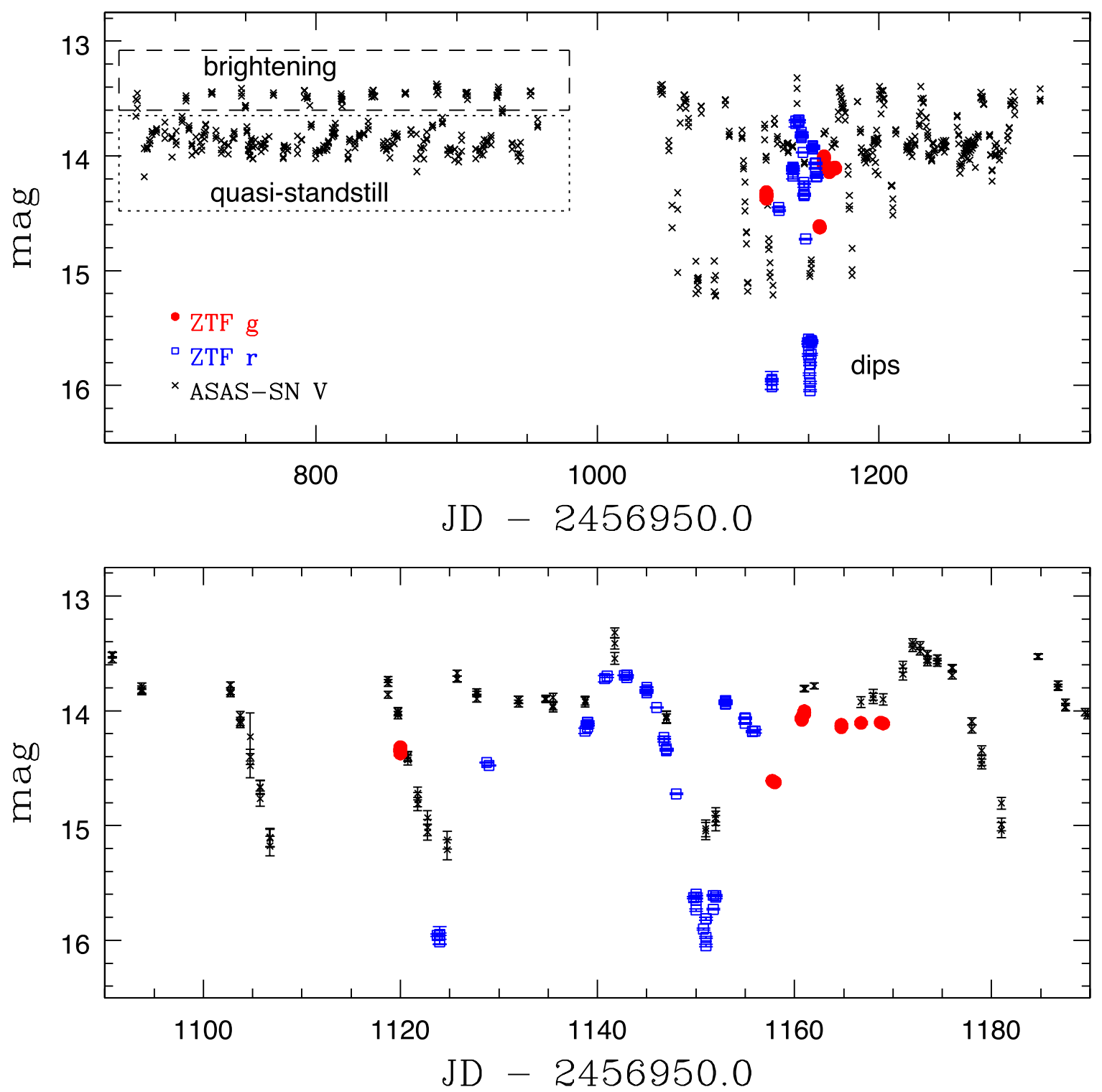

Figure 1. The ZTF $g$-band (red), $r$-band (blue), and ASAS-SN $V$-band (black) light curves of HO Pup. The top panel shows a portion of the light curve across $\sim 600$ days, together with terminologies that are used in this work to describe its features: quasi-standstills (data points in the dotted box) are part of the light curves with magnitudes close to the mean value; brightenings are data points (in the dashed box) showing a brightening of $\sim 0.5$ mag; and dips are events that fade by more than $\sim 1$ mag from the mean magnitude. Several fading events with dips of $\sim 2.5$ mag were observed recently. This epoch with a number of deep-dip events is enlarged in the bottom panel. Consistency of the light curves seen in both ZTF and ASAS-SN observations ruled out the possibility that such dips were due to instrumental effects.

photometric variation, known as an IW And-type star. The stars that display IW And-type phenomena represent a new subclassification of Z Cam stars (Kato 2019). The characteristics of IW And-type stars include eclipsing-like transients that happen once in a while, in addition to repeated brightening events seen in their light curves. Similar kinds of dip events followed by the outbursts or brightenings were also seen in several CVs. Unlike the deep dips of HO Pup, where it loses $90 \%$ of its starlight, other CVs tend to reduce by only about $50 \%$ of their outburst brightness (Mason \& Howell 2016; Schlegel \& Honeycutt 2019). At most, a reduction of $80 \%$ of the outburst brightness can be seen in the case of KIC 9406652 (Gies et al. 2013). Besides the prototype DN, IW And, in the literature several DNe such as IM Eri, FY Vul, V507 Cyg, ST Cha, V513 Cas, and KIC 9406652 have been classified as IW And-type stars, and the recent development of optical monitoring advances their identification (see Kimura et al. 2020a, 2020b, and reference therein). ${ }^{11}$ HO Pup could be an additional member of the IW And-type stars (Kimura et al. 2020a, see also vsnet-chat 8162 from VSNET Collaboration).

Given the ambiguous nature of HO Pup (as either a Be star or an IW And-type star), we collected its light-curve data as much as possible from archival catalogs, some ongoing surveys, and new dedicated observations. These collections of light curves are presented in Section 2. In addition to lightcurve data, we have also made spectroscopic and polarimetric observations of HO Pup, described further in the same section.

\footnotetext{
${ }^{11}$ More candidates for IW And-type stars can be found in VSNET Collaboration (http://ooruri.kusastro.kyoto-u.ac.jp/pipermail/vsnet-chat/). For example, V526 Ori (vsnet-chat 8474), MGAB-V1252 (vsnet-chat 8101), USNO-A2.0 1275-09782989 (vsnet-chat 8432), RX J1831.7+6511 (vsnet-chat 24230), V2837 Ori (vsnet-chat 23538), LN UMa (vsnet-chat 24347), and EZ Vul (vsnet-chat 8266).
} 

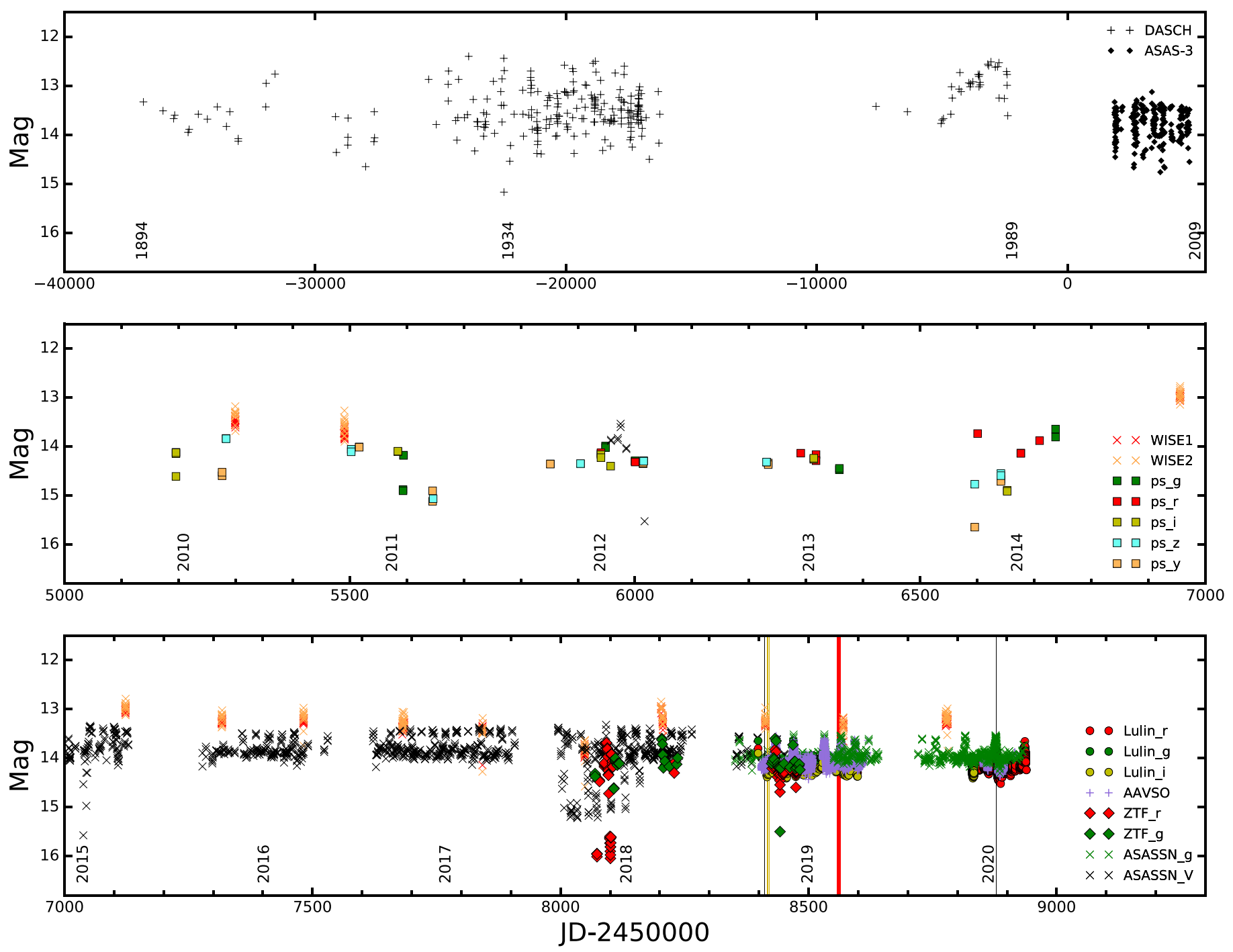

Figure 2. The light curve of HO Pup over more than a century from optical to mid-infrared, consisting of data from AAVSO, ASAS-3, ASAS-SN, DASCH, Lulin (with SLT), Pan-STARRS (PS), WISE, and ZTF. Each data point (for clarity, error bars are ignored) is noted with data source and filters accordingly. The times of follow-up spectroscopic and polarimetric observations are marked with vertical lines containing LOT/TRIPOL2 (yellow line in 2018), BOAO/BOES (blue line in 2018), CFHT/ESPaDOnS (red line in 2019), and P200/DBSP (black line in 2020). More details can be seen in Figure 6 with a focus on the individual duration.

Analysis and results based on the observations are presented in Section 3 , in which we also present the first emission-line spectra of HO Pup-confirming its emission-line nature. In Section 4, we discuss the scenarios for the observed unusual light-curve behavior of HO Pup, and we summarize our findings in Section 5.

\section{Observations and Data}

Time-series photometric data ranging from optical to infrared for HOPup were collected from various survey catalogs, including the ZTF, the ASAS-SN, the Digital Access to a Sky Century@ Harvard (DASCH, Grindlay et al. 2012), the third phase of the All Sky Automated Survey (ASAS-3, Pojmanski 2002), the Panoramic Survey Telescope and Rapid Response System $3 \pi$ survey (Pan-STARRS, Kaiser et al. 2010; Chambers et al. 2016), the Wide-field Infrared Survey Explorer (WISE, Cutri et al. 2012), and observations available via the American Association of Variable Star Observers (AAVSO). These light-curve data were supplemented with dedicated observations taken at the Lulin Observatory in Taiwan. All of
Table 1

Collected Light Curves with 28,700 Data Points for HO Pup

\begin{tabular}{lcccc}
\hline \hline MJD & Mag & Uncertainty & Band & Source \\
\hline 13146.3190 & 13.330 & 0.100 & $B$ & DASCH \\
13929.2218 & 13.510 & 0.170 & $B$ & DASCH \\
14353.0697 & 13.670 & 0.170 & $B$ & DASCH \\
14393.0297 & 13.600 & 0.170 & $B$ & DASCH \\
14927.3884 & 13.950 & 0.120 & $B$ & DASCH \\
14972.2725 & 13.890 & 0.160 & $B$ & DASCH \\
15336.3097 & 13.580 & 0.150 & $B$ & DASCH \\
15697.3473 & 13.680 & 0.150 & $B$ & DASCH \\
16093.2480 & 13.430 & 0.090 & $B$ & DASCH \\
16458.2944 & 13.830 & 0.180 & $B$ & DASCH \\
$\ldots$ & $\ldots$ & $\ldots$ & $\cdots$ & $\cdots$ \\
\hline
\end{tabular}

(This table is available in its entirety in machine-readable form.) 
Table 2

Summary of Light-curve Data

\begin{tabular}{|c|c|c|c|c|c|c|c|c|}
\hline Band & Database & $N$ & MJD (start) & MJD (end) & UT Date (start) & UT Date (end) & Mean Mag. & Std. \\
\hline$B$ & DASCH & 260 & $13,146.3$ & $47,616.4$ & 11-14-1894 & 03-31-1989 & 13.472 & 0.463 \\
\hline$g$ & ZTF & 54 & $58,107.4$ & $58,482.5$ & $12-20-2017$ & $12-30-2018$ & 14.136 & 0.288 \\
\hline$g$ & ASAS-SN & 937 & $58,220.2$ & $58,933.2$ & 04-12-2018 & $03-25-2020$ & 13.954 & 0.130 \\
\hline$g$ & Lulin/SLT & 313 & $58,397.9$ & $58,832.7$ & $10-06-2018$ & $12-15-2019$ & 14.128 & 0.089 \\
\hline$g$ & Pan-STARRS & 12 & $55,593.4$ & $56,737.3$ & 02-01-2011 & 03-21-2014 & 14.261 & 0.382 \\
\hline$v$ & ASAS-3 & 217 & $51,874.2$ & $54,862.2$ & $11-26-2000$ & 01-31-2009 & 13.793 & 0.310 \\
\hline$v$ & ASAS-SN & 960 & $55,957.4$ & $58,450.6$ & 01-31-2012 & $11-28-2018$ & 13.860 & 0.324 \\
\hline$v$ & AAVSO & 10890 & $58,404.3$ & $58,915.2$ & $10-13-2018$ & 03-07-2020 & 14.094 & 0.134 \\
\hline$r$ & ZTF & 494 & $58,073.3$ & $58,476.4$ & $11-16-2017$ & $12-24-2018$ & 14.283 & 0.311 \\
\hline$r$ & Lulin/SLT & 13786 & $58,397.9$ & $58,938.6$ & $10-06-2018$ & $03-30-2020$ & 14.190 & 0.099 \\
\hline$r$ & Pan-STARRS & 10 & $55,, 940.4$ & $56,709.3$ & 01-14-2012 & $02-21-2014$ & 14.109 & 0.175 \\
\hline$i$ & Lulin/SLT & 309 & $58,397.9$ & $58,832.7$ & 10-06-2018 & $12-15-2019$ & 14.293 & 0.067 \\
\hline$i$ & Pan-STARRS & 13 & $55,195.4$ & $56,652.5$ & $12-30-2009$ & $12-26-2013$ & 14.332 & 0.292 \\
\hline$z$ & Pan-STARRS & 14 & $55,283.3$ & $56,, 641.5$ & $03-28-2010$ & $12-15-2013$ & 13.311 & 0.336 \\
\hline$y$ & Pan-STARRS & 15 & $55,276.2$ & $56,641.5$ & $03-21-2010$ & $12-15-2013$ & 14.327 & 0.423 \\
\hline W1 & WISE & 208 & $55,298.7$ & $58,780.5$ & 04-12-2010 & $10-24-2019$ & 13.338 & 0.249 \\
\hline $\mathrm{W} 2$ & WISE & 208 & $55,298.7$ & $58,780.5$ & $04-12-2010$ & $10-24-2019$ & 13.280 & 0.273 \\
\hline
\end{tabular}

these light curves are merged in Figure 2 and listed in Table 1, which cover years from 1894 to 2020 . We also summarize the light-curve data in Table 2. Following the terminologies used to describe the light curves of IW And-type stars (Kimura et al. 2020a), definitions of the main features exhibited in the light curves, such as dips, brightenings, and quasi-standstills, are demonstrated in the upper panel of Figure 1. Spectroscopic observations with low and high spectral resolutions were conducted by P60/SEDM, BOAO/BOES, CFHT/ESPaDOnS, and P200/DBSP (all acronyms defined in Section 2.2). Additionally, polarization was measured on four different nights in late October 2018 using the TRIPOL2 instrument installed at the Lulin Observatory.

\subsection{Optical and Infrared Light-curve Data}

ZTF is a northern-sky synoptic survey project $\left(\delta>-30^{\circ}\right)$ carried out by the $1.2 \mathrm{~m}$ Samuel Oschin Telescope at the Palomar Observatory. With a large-field-of-view mosaic CCD camera ( $47 \mathrm{deg}^{2}$ with 1 !" 0 pixel scale), the Galactic plane can be scanned once a night with $g$ and/or $r$ filter. For HO Pup, 54 and 494 good-quality measurements were taken in the $g$ and $r$ bands, respectively, between 2017 December and 2018 December.

ASAS-SN data are taken by a couple of quadruple telescopes located in both hemispheres. The mounted cameras have a $4.5 \mathrm{deg}^{2}$ field of view with a pixel scale of 7 !" 8 . This survey provides us the longest-time baseline from 2012 to 2020, which contains $960 \mathrm{~V}$-band and $937 \mathrm{~g}$-band measurements.

The AAVSO responded quickly to get involved with the monitoring of HO Pup in the $V$ band, right after our report of this extraordinary event (see bottom panel of Figure 1) to the ZTF community. On 2018 October 13, the AAVSO began to collect data with extremely good coverage in the time domain, using an ML16803 CCD camera with a pixel scale of 2".06 equipped on a $0.4 \mathrm{~m}$ telescope at the Remote Observatory Atacama Desert (ROAD; Hambsch 2012) in San Pedro de Atacama, Chile. Every clear night, ROAD monitored HO Pup for more than $5 \mathrm{hr}$ with an average of 50 measurements, resulting in a total of 10,890 photometric data points. The typical uncertainty of the measurements is about 0.02 mag.
Here we used 0.15 mag as our quality threshold after testing several values.

Pan-STARRS used five filters, ${ }^{12} g_{p 1}, r_{p 1}, i_{p 1}, z_{p 1}$, and $y_{p 1}$, to survey the sky. It has a wide-field $7 \mathrm{deg}^{2}$ mosaic camera with a pixel scale of 0 !'26, mounted on the dedicated $1.8 \mathrm{~m}$ PanSTARRS telescope, which is located at the Haleakale Observatory in Hawaii. HO Pup was observed over a full five-year time span (2009-2014). In contrast to ASAS-SN or AAVSO, there are only a small number of observations. Two significant dips $(m>15.5 \mathrm{mag})$ were also recorded by PanSTARRS.

The $0.4 \mathrm{~m}$ SLT telescope, located at the Lulin Observatory in Taiwan, was used to perform near-simultaneous gri-band and intensive $r$-band monitoring in follow-up observations of HO Pup. Together with the equipped Apogee U42 CCD, SLT images have a pixel scale of 0 ." 79 . We used 77 reference stars located within 0.5 of $\mathrm{HO}$ Pup to perform differential photometry and calibrated to the Pan-STARRS catalog. The SLT data provide significant support to the variability investigation on a short timescale and monitoring of the color variation in the optical because the multiband data from the optical surveys mentioned earlier were not taken simultaneously or nearly simultaneously (hence no color information).

WISE is an all-sky survey mission that mapped the entire sky at 3.4, 4.6, 12, and $22 \mu \mathrm{m}$ (hereafter referred as W1, W2, $\mathrm{W} 3$, and W4) with spatial resolutions of 6!" 1 and 6!" 4 in W1 and W2 bands (Mainzer et al. 2011), respectively. The nearEarth object WISE (NEOWISE) project is particularly designed to hunt for asteroids using the W1 and W2 bands, and it provides infrared (IR) data over nine years from 2010 April to 2019 October. The cadence of the WISE observations is about twice a year; each observation includes about a dozen measurements over one day.

Finally, we considered the utility of the DASCH and ASAS3 light-curve data, but they were not used in subsequent analysis. The DASCH project has digitized photometric measurements from nearly 500,000 glass plates over 100 years. With a quality cut of $0.2 \mathrm{mag}, 260$ Johnson $B$-band magnitudes were selected for HO Pup from 1894 up to 1989 as historical records. One possible dip is included (see

\footnotetext{
${ }^{12}$ For simplicity, we refer to them as $g, r, i, z$, and $y$ in the rest of this paper.
} 
Section 3.2). In the case of ASAS-3 data (Pojmanski 1997, 2002), we excluded them from the analysis because of the associated large pixel scale $\left(\sim 15^{\prime \prime}\right)$. Therefore, the issue of blending is unavoidable at the location of HO Pup. Nevertheless, we extracted $217 \mathrm{~V}$-band light curves (grades A and B only) from the ASAS-3 archive. These light-curve data were measured using the smallest ASAS-3 aperture size (Pojmanski et al. 2005, 2 pixels; corresponding to MAG_0 in ASAS-3 catalogs).

\subsection{Spectroscopic Data}

Soon after the 2.5 mag dips found by ZTF in late 2017, we collected spectra of HO Pup based on the observations carried out in 2018 by P60/SEDM and BOAO/BOES, in 2019 by CFHT/ESPaDOnS, as well as in 2020 by P200/DBSP. Due to the nature of queue observations for these telescopes and instruments, none of the spectra were taken during the $2.5 \mathrm{mag}$ dip event of HOPop, which did not occur again after mid-2018.

The Spectral Energy Distribution Machine (SEDM, BenAmi et al. 2012; Ritter et al. 2014; Blagorodnova et al. 2018; Rigault et al. 2019) is a low-resolution integral field unit spectrograph, mounted on the robotic P60 telescope at the Palomar Observatory (Cenko et al. 2006), providing efficient follow-up observations. The dispersions on the red side and blue side are 35 and $17.4 \AA$ per pixel, respectively. Queued observations of SEDM were carried out multiple times between 2018 October 19 and 2018 November 7, but only spectra from four nights were usable. The data were automatically reduced using the dedicated SEDM reduction pipeline (Rigault et al. 2019). The low-resolution P60/SEDM spectra can only be used to identify $\mathrm{H} \alpha$ emission lines, hence they were excluded from this work.

We have also obtained an optical spectrum of HO Pup using the Bohyunsan Optical Echelle Spectrograph (BOES, Kim et al. 2002) in long-slit mode, mounted on the $1.8 \mathrm{~m}$ Optical Telescope at the Bohyunsan Optical Astronomy Observatory (BOAO) in South Korea, on 2018 October 20. The observation was conducted using the grating $300 \mathrm{~V}$ with $3^{\prime \prime}$ slit width under 1!. 8 seeing, giving a spectral resolution of $R \sim 1200$. The wavelength coverage is $3500-7000 \AA$ and the exposure time is 40 minutes. We follow the standard procedures of data reduction using IRAF, ${ }^{13}$ e.g., flat-fielding, wavelength calibration with $\mathrm{FeNeArHe}$ lamp, and flux calibration with the standard star G191-B2B. The normalized spectrum is shown in the bottom panel of Figure 4. The $\mathrm{H} \alpha$ emission line is clearly detected with an equivalent width (EW) of $\sim-4.3 \AA$, estimated using the Astrolib PySynphot package (STScI Development Team 2013). Due to low signal-to-noise ratio below $5000 \AA$ of the spectrum, we cannot confirm the detailed spectral type of HO Pup.

To further investigate the $\mathrm{H} \alpha$ emission variability, we took high-resolution echelle spectra of HO Pup using the ESPaDOnS (Echelle Spectropolarimetric Device for the Observation of Stars) mounted on the $3.6 \mathrm{~m}$ Canada-France-Hawaii Telescope (CFHT), with a spectral resolution of $R \sim 68,000$. The wavelength coverage is $3750-10400 \AA$ at approximately $0.007 \AA /$ pixel, which contains 40 grating orders. With an

\footnotetext{
${ }^{13}$ IRAF is distributed by the National Optical Astronomy Observatory, which is operated by the Association of Universities for Research in Astronomy, Inc. under cooperative agreement with the National Science Foundation.
}
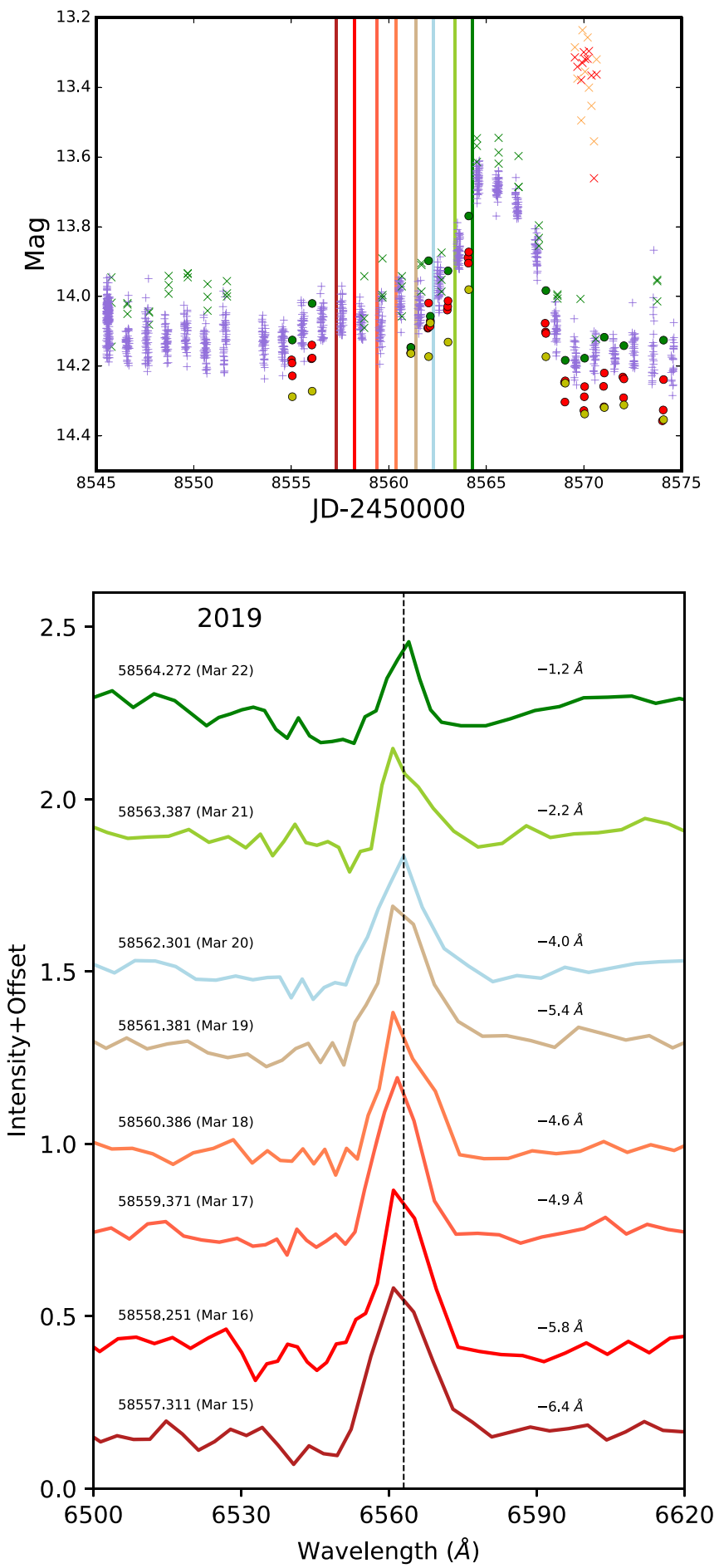

Figure 3. Top panel is the same as Figure 2, except for the epoch with CFHT/ ESPaDOnS observations. The bottom panel shows the CFHT/ESPaDOnS spectra of HO Pup taken from 2019 March 15 to 22, centered on the $\mathrm{H} \alpha$ line (vertical dashed line). To improve the signal-to-noise ratio in the plot, the spectra were rebinned with a resolution of $\delta \lambda \sim 3 \AA$. Values on the right of each spectrum are the measured EW of the $\mathrm{H} \alpha$ line, derived using the Astrolib PySynphot package (STScI Development Team 2013).

exposure time of $2400 \mathrm{~s}$ for spectroscopy (sky+star mode) and $4800 \mathrm{~s}$ for spectropolarimetry (both were arranged by the CFHT queue observers), HO Pup was nightly observed from 2019 March 15 to 22 under good seeing conditions $\left(\lesssim 1^{\prime \prime}\right)$. CFHT provided a set of fully reduced spectra from Libre Esprit, an automatic ESPaDOnS reduction package/pipeline 

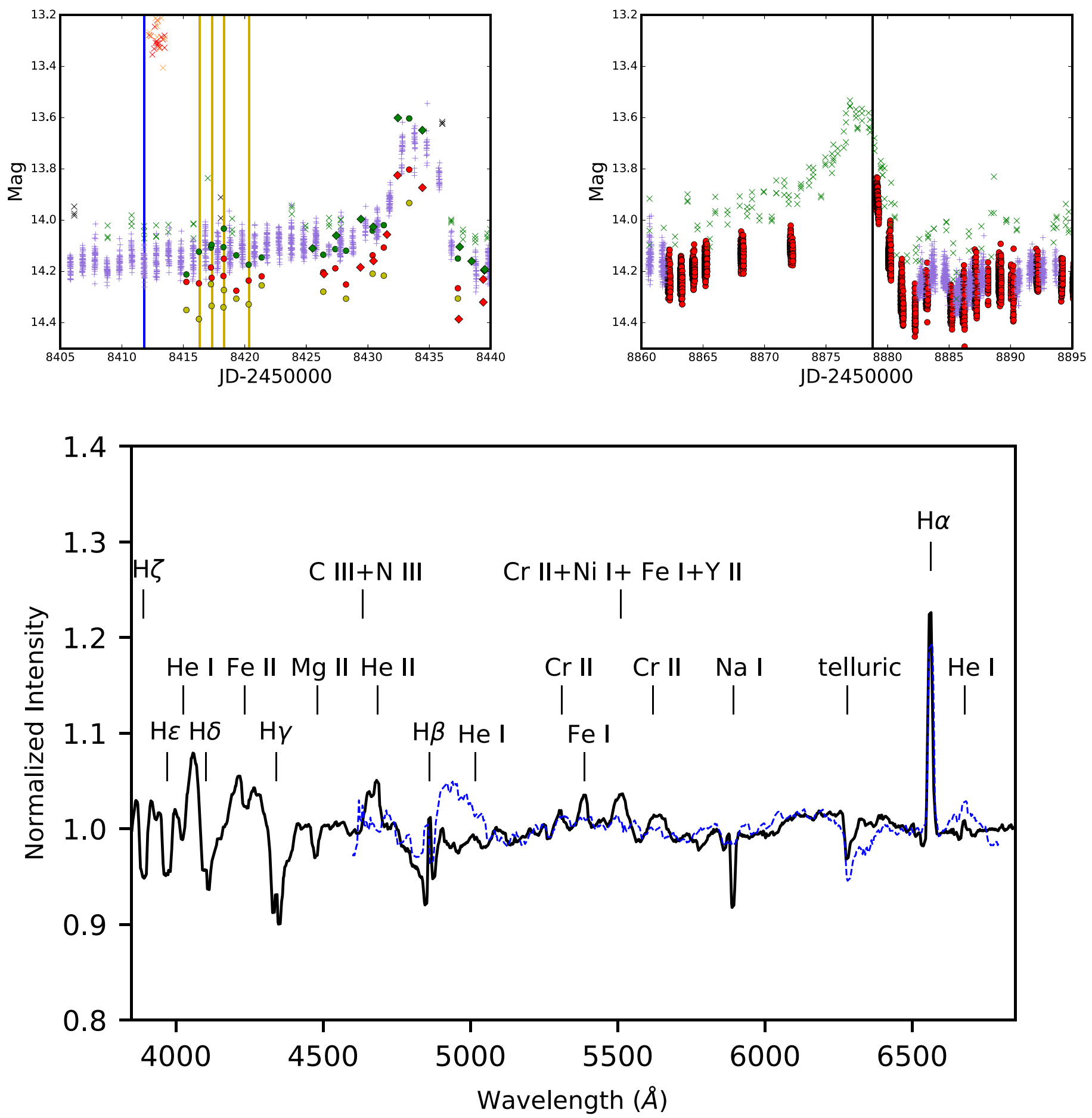

Figure 4. Top panels are the same as Figure 2, except for the epoch with BOAO/BOES (blue line) and LOT/TRIPOL2 (yellow lines, see Table 3) observations in the top left panel. The top right panel is for the epoch with P200/DBSP spectroscopic observation (black line), taken right after the photometric maximum of one of the brightening events. The bottom panel shows the BOAO/BOES spectrum (dashed blue curve) and P200/DBSP spectrum (solid black curve) resulting from a normalization using a fifth-order polynomial. Both the $\mathrm{H} \alpha$ and $\mathrm{H} \beta$ lines are obviously seen in these spectra with either low or medium resolution, hence the emission nature of HO Pup was essentially confirmed. The spectral characteristics at the phase of brightening, including hydrogen Balmer lines, as well as helium, iron, nickel, sodium, and chromium lines, are identified.

(Donati et al. 1997, 2007). One of the brightenings or brightening events from $14.1 \mathrm{mag}$ to $13.6 \mathrm{mag}$ was fortunately well observed during our observing runs. As shown in Figure 3, all $\mathrm{H} \alpha$ emission lines are clearly seen, despite the fact that parts of the continuum were barely observed.

Finally, a single $600 \mathrm{~s}$ spectrum was obtained using the Palomar 200 inch Hale Telescope (P200) with the Double-
Beam Spectrograph (DBSP, Oke \& Gunn 1982) on 2020 January 30. A $2^{\prime \prime}$ slit was used with the $600 / 4000$ grating in the blue arm and the $316 / 7500$ grating in the red arm, providing $R \sim 1500$. A wavelength calibration spectrum was taken directly after the science spectrum. A spectrum of a fluxstandard star was obtained at the beginning and end of the night. The data were reduced using the pipeline developed by 
Table 3

Polarization Values of HO Pup

\begin{tabular}{lcccc}
\hline \hline Date & $\begin{array}{c}P_{r} \\
(\%)\end{array}$ & $\begin{array}{c}\theta_{r} \\
(\mathrm{deg})\end{array}$ & $\begin{array}{c}P_{i} \\
(\%)\end{array}$ & $\begin{array}{c}\theta_{i} \\
(\mathrm{deg})\end{array}$ \\
\hline 2018 October 24 & $0.7 \pm 0.5$ & $44.4 \pm 22.5$ & $0.9 \pm 0.2$ & $45.8 \pm 34.1$ \\
2018 October 25 & $0.5 \pm 0.5$ & $135.8 \pm 28.6$ & $0.5 \pm 0.5$ & $132.9 \pm 28.6$ \\
2018 October 26 & $1.5 \pm 0.2$ & $28.1 \pm 11.3$ & $0.8 \pm 0.3$ & $30.8 \pm 42.4$ \\
2018 October 28 & $0.0 \pm 0.5$ & $25.6 \pm 28.6$ & $0.7 \pm 0.7$ & $128.3 \pm 28.6$ \\
\hline
\end{tabular}

Bellm \& Sesar (2016). ${ }^{14}$ Using the pipeline, we performed the standard bias corrections, flat-field corrections, wavelength calibration, and flux calibration. We also automatically combined the red and blue spectra using this pipeline. The reduced spectrum is displayed in the lower panel of Figure 4, in which the $\mathrm{H} \alpha \mathrm{EW}$ value is $\sim-4.0 \AA$, also estimated using the Astrolib PySynphot package.

\subsection{Polarimetric Data}

In addition to the photometric and spectroscopic observations, we also took polarimetric data by using the TRIPOL2 (second generation of the Triple Range Imager and Polarimeter) instrument (Sato et al. 2019), equipped on the Lulin One-meter Telescope (LOT), in 2018 October. TRIPOL2 can simultaneously take polarization images in Sloan $g^{\prime}, r^{\prime}$, and $i^{\prime}$ bands with the half-wave plate rotating to four angles: $0^{\circ}, 22^{\circ} .5$, $45^{\circ}$, and $67^{\circ} .5$. We measured the flux at each angle using aperture photometry following standard reduction procedures, and the Stokes parameters $(I, Q$, and $U$ ) were then derived. The polarization percentage $P=\sqrt{Q^{2}+U^{2}} / I$ and the position angle $\theta=0.5 \arctan (U / Q)$ can be calculated from these Stokes parameters with a typical accuracy of $\Delta P \lesssim 0.3 \%$. Polarimetric data of HO Pup were well obtained in $r$ and $i$ bands (but it were too faint in the $g$ band), as reported in Table 3. We have also observed a number of unpolarized and polarized standard stars (Schmidt et al. 1992) to calibrate the instrumental polarization and angle offset (Sato et al. 2019). Based on the observations of these standard stars, we found that the performance of TRIPOL2 was very stable during those nights for both the measurements of polarization percentages (using both polarized and unpolarized standard stars) and the polarization position angles (using the unpolarized standard stars); for more details, see Huang (2019). To reduce the influence by sky conditions, we also require that each of these four measurements has at least five sets of images with nearly the same count at each angle. This ensures that the polarization measurements are reliable from our nightly observations.

\section{Results}

\subsection{An Emission-line Object}

As mentioned in the Introduction, HO Pup could be considered as a Be-star candidate or an IW And-type DN, without any emission-line or even spectral observations reported in the literature. With our spectroscopic follow-up observations, the $\mathrm{H} \alpha$ emission line is clearly seen in each of our observations including the spectra from $\mathrm{BOAO} / \mathrm{BOES}$, CFHT/ESPaDOnS, and P200/DBSP (as shown in Figures 3 and 4). The $\mathrm{H} \alpha$ emission EW is weak, e.g., $\sim-4.5 \AA$ as measured in the BOAO/BOES spectrum. Therefore, in addition to the known GCAS or IW And-type light-curve variation, HO Pup is now confirmed as an emission-line object.

Beyond $\mathrm{H} \alpha$ lines, other prominent hydrogen, helium, and metal features can be easily identified. During the brightening phase, HO Pup shows Balmer lines, from $\mathrm{H} \beta$ to $\mathrm{H} \zeta$, which are superposed on weak emission lines (see Figure 4). The helium features show absorption lines He I $4026 \AA$ and $4471 \AA$, and emission lines He I $5016 \AA$, $6678 \AA$, and He II $4713 \AA$ A. Several metal absorption features can be identified, such as Fe II $4233 \AA, \mathrm{Mg}$ II $4481 \AA$, and Na I doublet $5889 \AA$ and $5893 \AA$; other emission features include Cr II $5311 \AA$ and $5620 \AA$, and Fe II $5387 \AA$. We also identified a few blended emission lines such as C III + N III 4634-4651 $\mathrm{A}$ (blended Bowen lines) and $\mathrm{CrII}+\mathrm{NiI}+\mathrm{Fe}$ I + Y II $5510 \AA$. Note that the spectral characteristics and EW variation of $\mathrm{H} \alpha$ emission for HO Pup will be discussed further in Section 4.

\subsection{The Dip Events}

Two 2.5 mag dips were observed in the ZTF $r$ and $g$ bands (13.5-16 mag; see Figure 1) in late 2017. During these

Table 4

Faintest Measurements of Each Dip Event

\begin{tabular}{|c|c|c|c|c|c|c|}
\hline$\overline{\mathrm{JD}}$ & Date & Surveys & Mag & Error & Band & $\overline{\text { Event No. }}$ \\
\hline $2,427,525.315$ & $1934 / 03 / 28$ & DASCH & 15.170 & 0.150 & $B$ & B1 \\
\hline $2,455,645.785$ & $2011 / 03 / 25$ & Pan-STARR & 15.119 & 0.005 & $\mathrm{y}$ & B2 \\
\hline $2,456,016.802$ & $2012 / 03 / 30$ & ASAS-SN & 15.526 & 0.07 & $v$ & B3 \\
\hline $2,456,596.153$ & $2013 / 10 / 30$ & Pan-STARR & 15.646 & 0.006 & $\mathrm{y}$ & B4 \\
\hline $2,457,038.010$ & $2015 / 01 / 15$ & ASAS-SN & 15.576 & 0.156 & $v$ & B5 \\
\hline $2,458,007.137$ & $2017 / 09 / 10$ & ASAS-SN & 15.017 & 0.194 & $v$ & E1 \\
\hline $2,458,021.849$ & $2017 / 09 / 25$ & ASAS-SN & 15.175 & 0.084 & $v$ & E2 \\
\hline $2,458,033.880$ & 2017/10/07 & ASAS-SN & 15.224 & 0.122 & $v$ & E3 \\
\hline $2,458,049.806$ & $2017 / 10 / 23$ & ASAS-SN & $>15.285$ & & $v$ & E4 \\
\hline $2,458,049.435$ & $2017 / 10 / 23$ & WISE & 14.581 & 0.174 & W2 & E4 \\
\hline $2,458,056.777$ & $2017 / 10 / 30$ & ASAS-SN & 15.178 & 0.087 & $v$ & E5 \\
\hline $2,458,074.053$ & $2017 / 11 / 16$ & ZTF & 15.958 & 0.078 & $r$ & E6 \\
\hline $2,458,074.771$ & $2017 / 11 / 17$ & ASAS-SN & 15.211 & 0.088 & $v$ & E6 \\
\hline $2,458,101.029$ & $2017 / 12 / 13$ & ZTF & 15.975 & 0.01 & $r$ & E7 \\
\hline $2,458,101.081$ & $2017 / 12 / 13$ & ASAS-SN & 15.05 & 0.074 & $v$ & E7 \\
\hline $2,458,130.982$ & $2018 / 01 / 12$ & ASAS-SN & 15.041 & 0.066 & $v$ & E8 \\
\hline $2,458,442.964$ & $2018 / 11 / 20$ & ZTF & 15.5 & 0.087 & $g$ & A1 \\
\hline
\end{tabular}




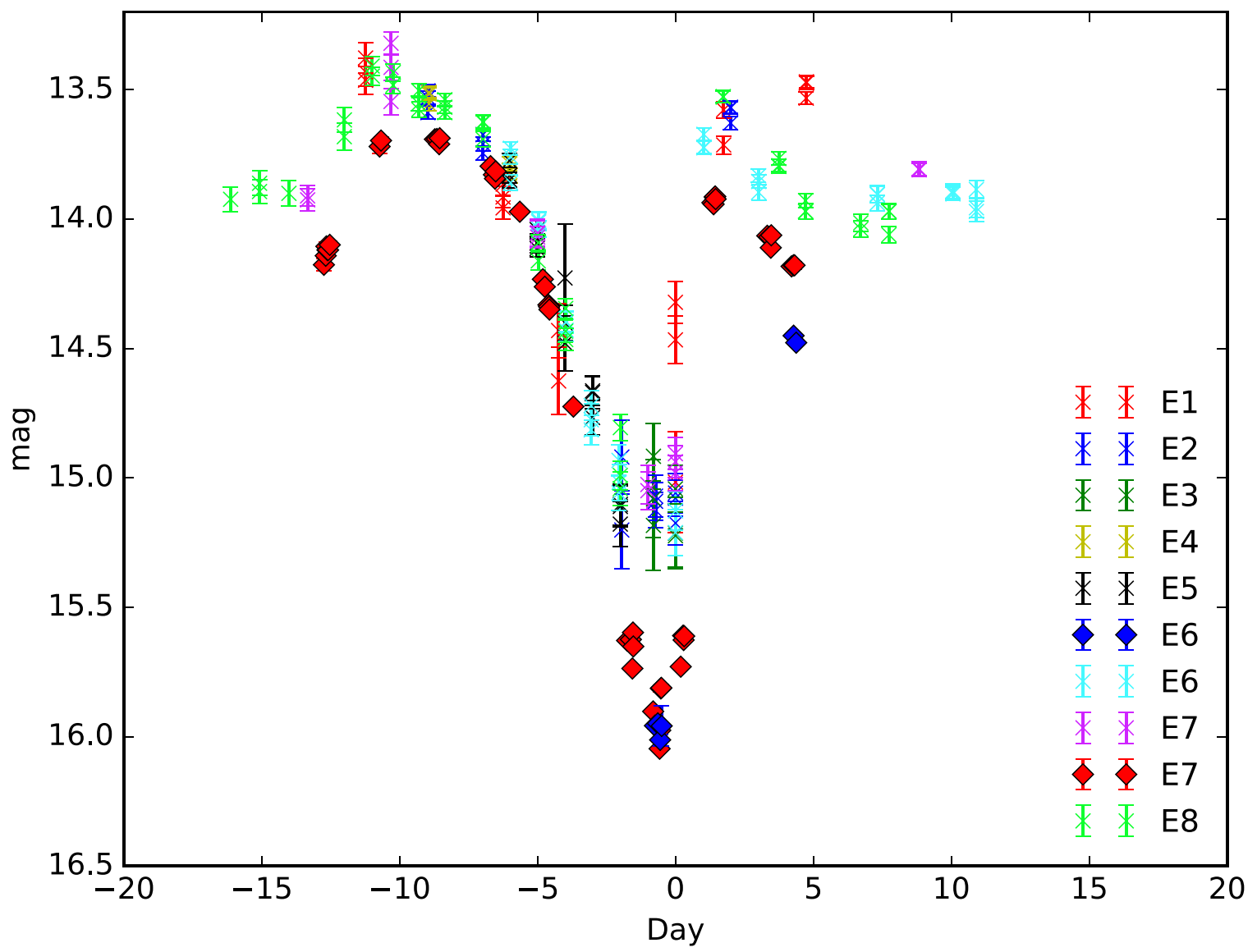

Figure 5. Dip events observed in the 2017-2018 season, where the diamonds and crosses represent the ZTF and ASAS-SN data, respectively. Two 2.5 mag dips, E6 and E7, are overplotted with six other similar dips-E1, E2, E3, E4, E5, and E8. We aligned these events using their own fading slopes, which have almost the same decreasing trend. With visual inspection, the minimum of each dip is chosen as day zero on the $x$-axis.

significant events, ASAS-SN also witnessed these two dips in the $V$ band. These dips observed in the two independent surveys are well matched in the time sequence. When examining the long-term light curve taken from ASAS-SN, in total there are eight dip events found between 2017 September and 2018 January, labeled as E1 to E8 in Table 4. Note that the dip event E4 was barely observed by ASAS-SN on 2017 October 23 (JD =2,458,049.806). Within half a day, WISE happened to catch the IR counterpart of the same event down to $14.58 \mathrm{mag}$ in the $\mathrm{W} 2$ band ( $\mathrm{JD}=2,458,049.435)$. Just like other dips in optical bands, the IR brightness drops significantly during this event when compared to the average brightness in the WISE W2 band (13.280 mag).

The E1-E8 dips have very similar shapes, especially their decreasing slopes. In Figure 5, we overplotted these events all together to show their similar shapes. Note that only the E6 and E7 events were found to drop by $\sim 2.5 \mathrm{mag}$, while the other events show drops in magnitude that range from $\sim 1$ to $\sim 2$ mag. This could be due to the issue of sampling the light curve around these events (i.e., the observations miss the epochs of the exact event minimum). However, these dip events occurred aperiodically in the range $\sim 7$ to $\sim 30$ days. Using the entire light-curve coverage from E1 to E8, the most likely event duration was found to be around 14.3 days, estimated using the method of phase dispersion minimization (PDM, Stellingwerf 1978).

\footnotetext{
${ }^{14}$ https://github.com/ebellm/pyraf-dbsp
}

In the early epochs from 2011 to 2015 , the dips B2 to B5 were individually recorded by Pan-STARRS or ASAS-SN, as seen in Table 4. A suspected dip, B1, was also recorded in the DASCH light curve around 1934. In addition, one more dip, A1, was caught by ZTF during the 2018-2019 season. Before A1 occurred (2018 November 20), we have four successful SEDM observations. Unfortunately, there is a long queue of observing requests on SEDM until the end of our proposed observation run on 2018 November 27. Thus, we did not have any further observation and missed the opportunity to catch spectroscopic observations of this dip.

\subsection{Semiregular 0.5 mag Brightening Events}

In addition to the dip events mentioned in the previous subsection, the long-term light curves of HO Pup also exhibit semiregular $\sim 0.5$ mag brightening events known as brightenings. These brightening events can been seen in Figure 2, for example, during the 2016 and 2017 seasons, which are better visualized in the upper panels of Figure 4. In Figure 6, we split the $\sim 7$ years of light curves of HO Pup into different segments. The $\sim 0.5 \mathrm{mag}$ brightening events are clearly showing in the light curves repeatedly with a duration between 21 and 61 days, as displayed in Figures 6(a)-(f).

An initial analysis suggested that these brightening events exhibit some periodicity, but no single period can be determined. Therefore, we estimated the periodicity on different segments of the light curves using the PDM approach. These segments are similar to those presented in Figure 6. We 

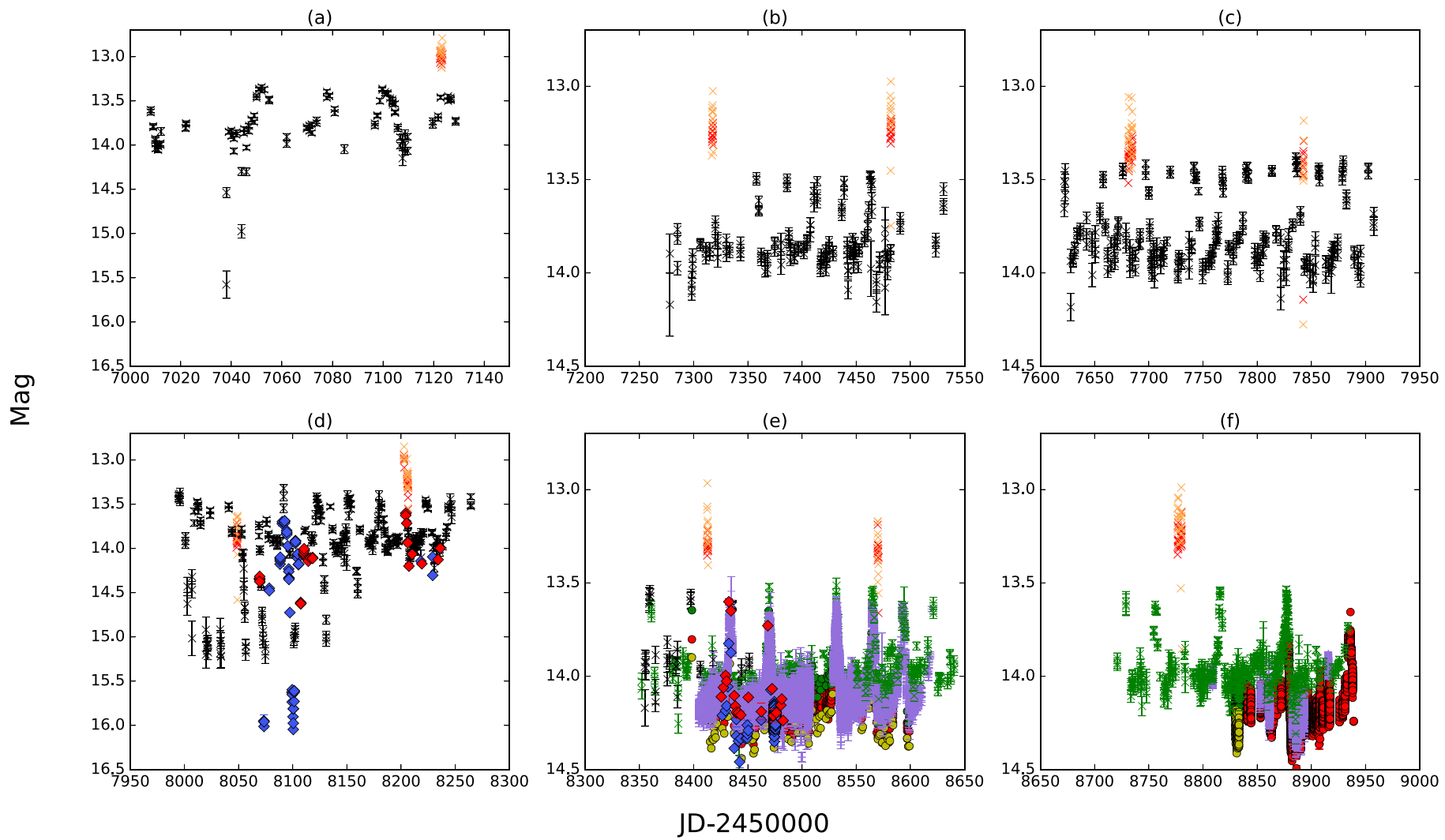

Figure 6. Same as Figure 2, but timescale enlarged for the different years of interest: (a) 2014-2015, (b) 2015-2016, (c) 2016-2017, (d) 2017-2018, (e) 2018-2019, and (f) 2019-2020.
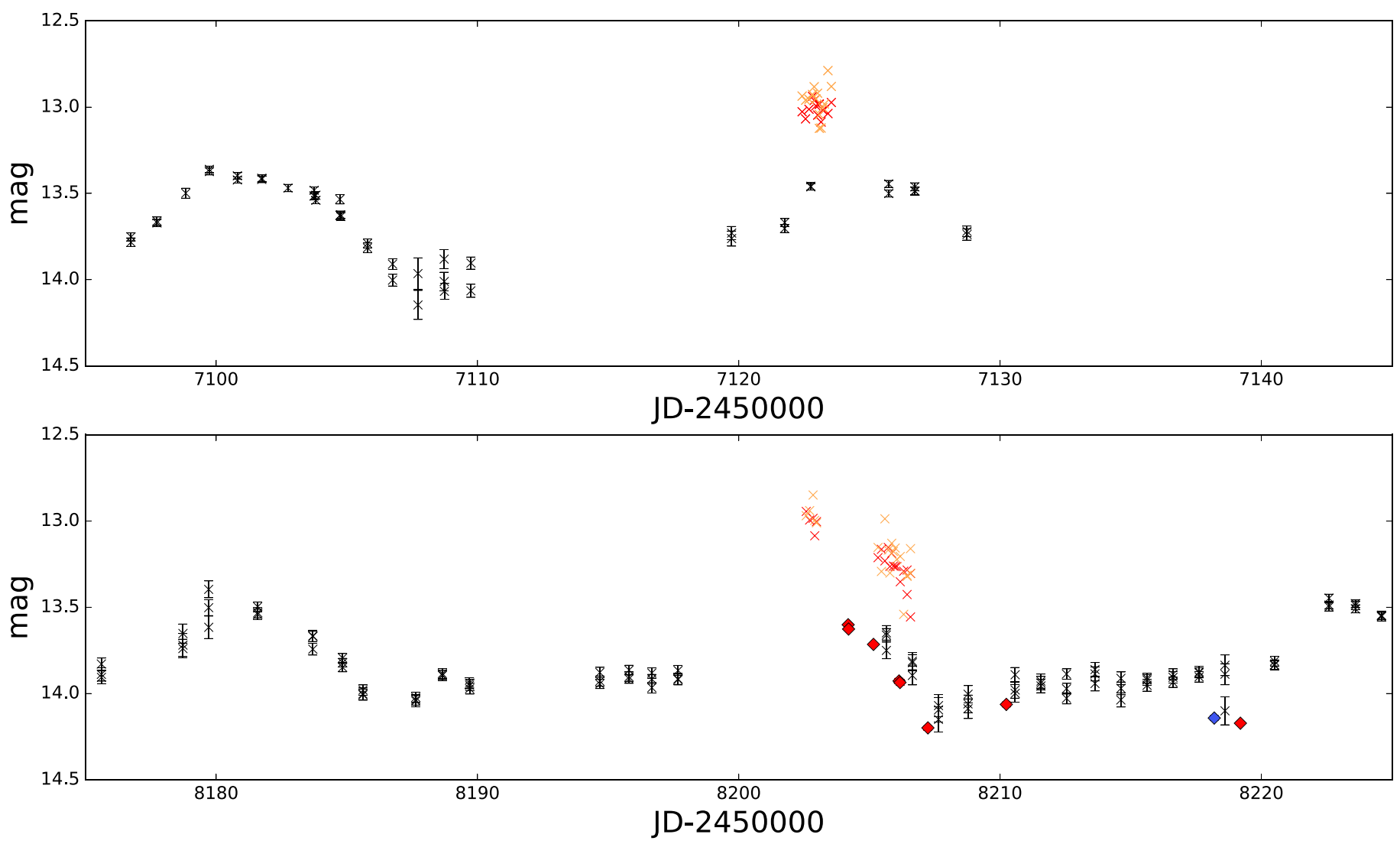

Figure 7. Same as Figure 2, but timescale enlarged for the two 0.5 mag brightening events as observed with the WISE data in 2015 (upper panel) and 2018 (lower panel). 

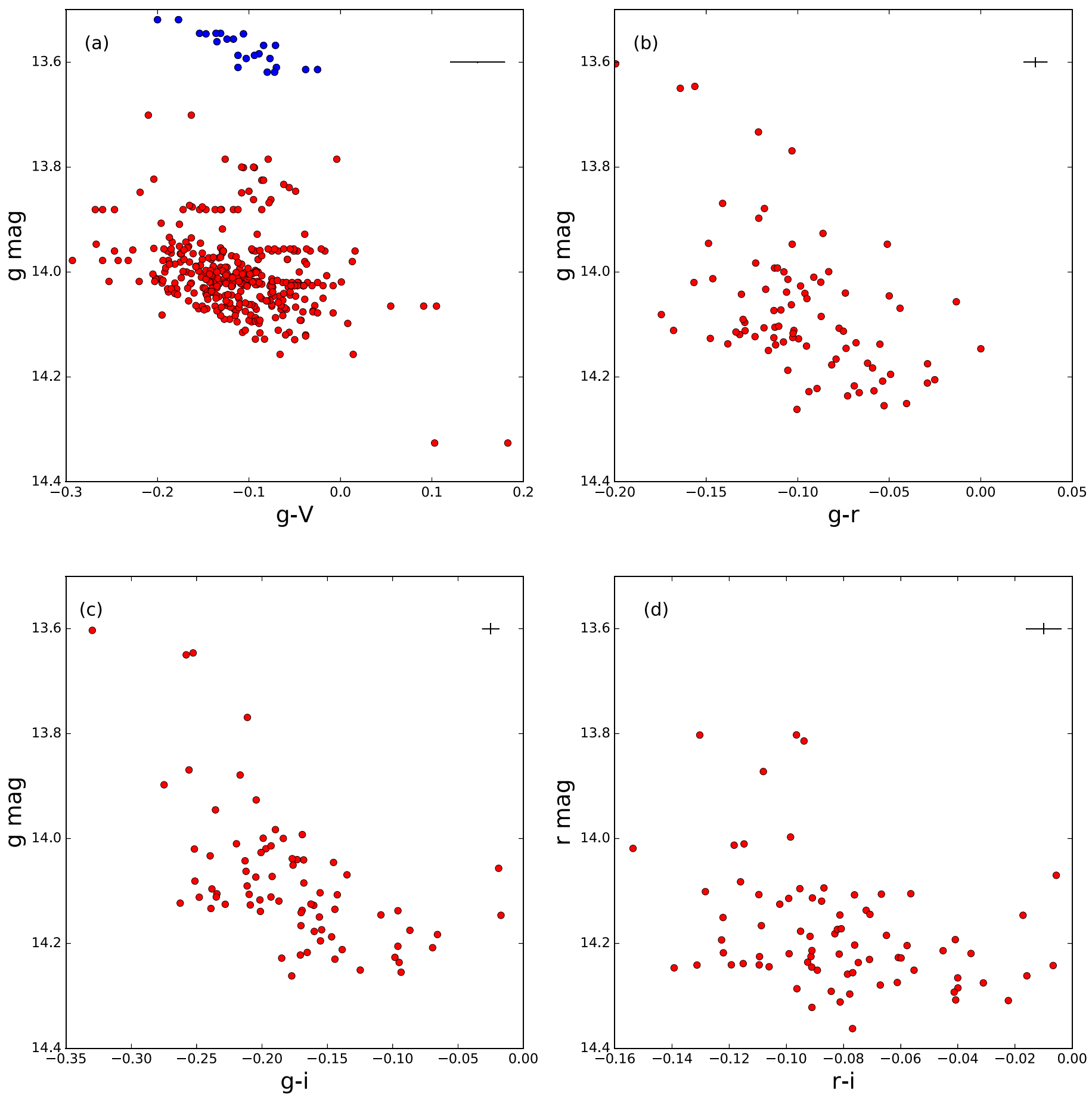

Figure 8. Color-magnitude diagrams of HO Pup. The representative error bars of colors and magnitudes are shown in the upper right corners of these four CMDs. The $g$ - and $V$-band data in panel (a) were taken from ASAS-SN and AAVSO, respectively. The intense observations from both surveys allow us to separate the data points into those that occurred at the $\sim 0.5 \mathrm{mag}$ brightening events (blue points) as discussed in Section 3.3, and those outside these events (red points). In panels (b)-(d), the gri-band data are based on the SLT observations taken at the Lulin Observatory. All of these CMDs show that HO Pup becomes bluer when it gets brighter.

derived mean periods of 25.9 days and 22.8 days for the lightcurve data in 2015-2016 (JD: 2,457,250-2,457,500) and 2016-2017 (JD: 2,457,500-2,457,950), respectively. For 2017-2018 (JD: 2,457,950-2,458,300), since the light curves are dominated by the dip events as shown in the previous subsection, we excluded this part of the light curves from our PDM analysis. In 2018-2019 (JD: 2,458,300-2,458,650), visual inspection suggested that the periodicity of the brightening events varied sporadically with longest and shortest periods of 37 and 26 days, respectively. Therefore we divided the light curves of 2018-2019 into three parts (in JD): $2,458,300-2,458,485,2,458,485-2,458,570$, and those later than $2,458,570$. The corresponding periods found by applying the multiband PDM analysis are $35.6 \pm 0.03,31.9 \pm 0.18$, and $26.8 \pm 0.34$ days, respectively. Finally, in the recent epoch 2019-2020 (JD: 2,458,720-2,458,933), the estimated period turns out to be $60.9 \pm 0.28$ days. It is worth pointing out that these repeatable brightening events resemble the light curves of IW And-type stars (Kimura et al. 2020a). 

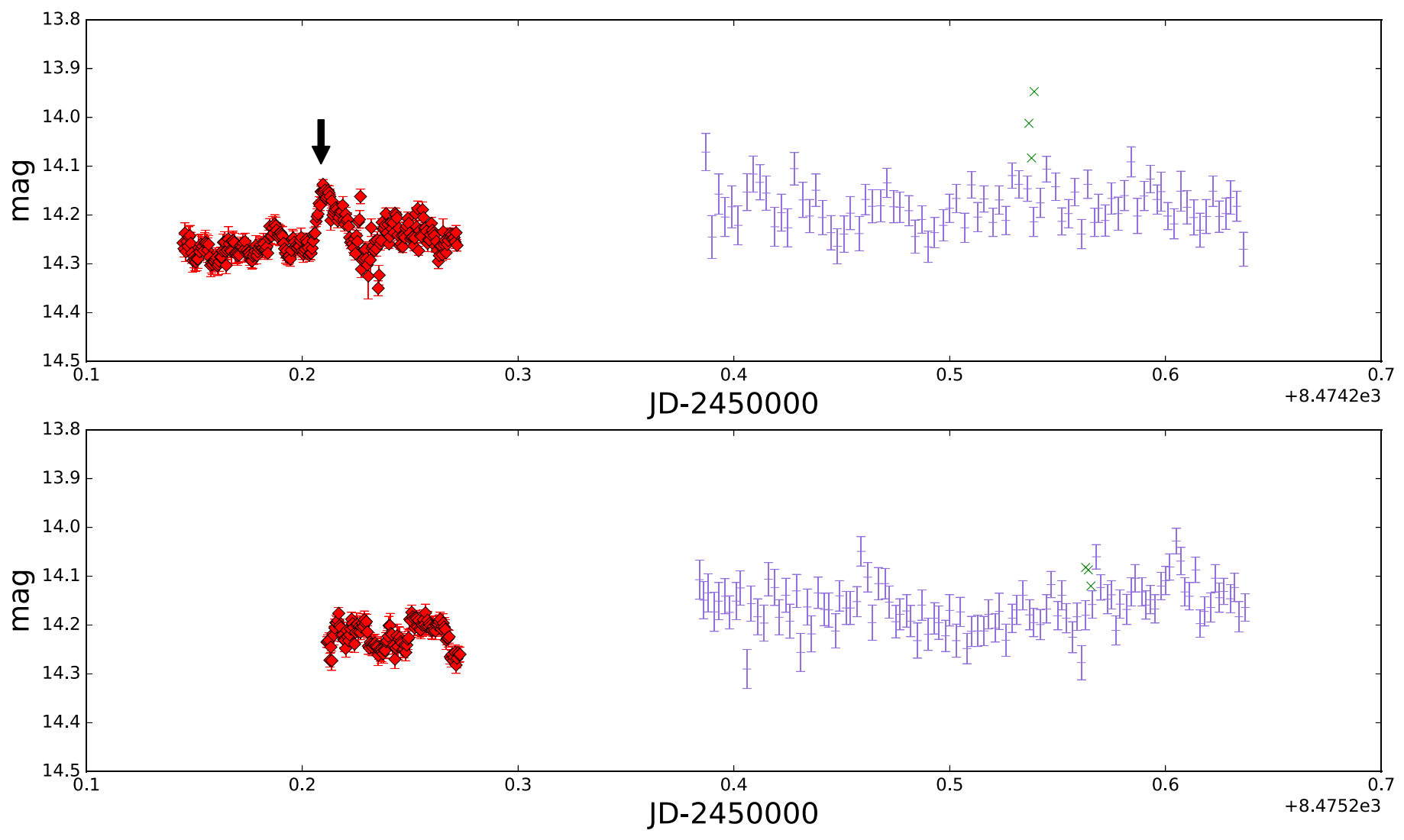

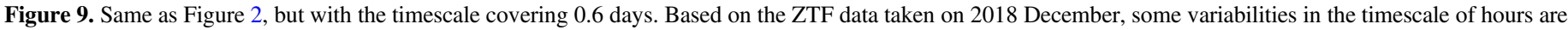

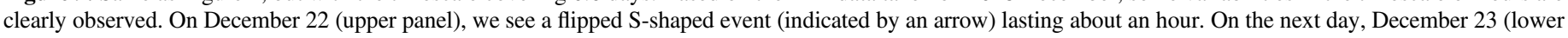

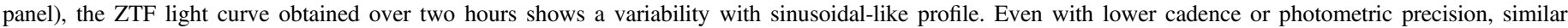
variations are also seen in the AAVSO light curve.

In addition to those in the optical, two brightening events were also observed in the mid-IR from WISE. As shown in Figure 7, these brightening events in the mid-IR were almost varying together with the optical ASAS-SN $V$-band light curve, suggesting that the entire continuum rises up during the brightening events.

For cases in which spectroscopic observations are possible, we have continuously taken spectra every night using CFHT/ ESPaDOnS before the optical light curve reached its maximum brightness in one of the brightening events that occurred in 2019 March (see Figure 3). We found that the strength, or equivalently the $\mathrm{EW}$, of the $\mathrm{H} \alpha$ line decreased while HO Pup became brighter, as shown in the spectra of the last two days in Figure 3.

\subsection{Color Variations}

We investigated the color variations of HO Pup using several measurements. To construct the $(g-V)$ color, we selected $g$ band data from ASAS-SN and $V$-band data from AAVSO. Each $g$-band point and its corresponding $V$-band point were observed within 5 minutes. Also, we used gri-band data of the SLT telescope at the Lulin Observatory to construct the $(g-r)$, $(r-i)$, and $(g-i)$ colors, where the gri-band images were always taken nearly simultaneously, e.g., within 5 minutes. To quantify the color variation with brightness, we plotted the magnitudes as a function of color (i.e., the color-magnitude diagram, CMD) in Figure 8. In all these optical colors, the blue data points correspond to the $\sim 0.5 \mathrm{mag}$ brightening events and the red data points were taken during the quasi-standstill phase.
As can be seen from Figure 8(a), the colors in these two phases cover very similar ranges. Furthermore, all of the colors present a trend such that they become bluer when HO Pup becomes brighter, especially for the $(g-i)$ colors shown in Figure 8(c).

\subsection{Variabilities on a Short Timescale}

In addition to the dip events and brightening events, as presented in Sections 3.2 and 3.3, respectively, short-term variabilities were also found in the light curves of HO Pup during its quasi-standstill phase. For example, in late 2018 December, ZTF performed continuous cadence observations on the Galactic plane that included HO Pup. Therefore, continuous $r$-band light curves taken within $2 \mathrm{hr}$ were available from ZTF on 2018 December 22 and 23, with 280 and 136 measurements, respectively (see Figure 9). An hour-scale sinusoidal variability with $\sim 0.1$ mag to $\sim 0.2$ mag amplitude was revealed in this set of ZTF light curves. Similar variability can also be seen in the AAVSO light curve, even though the cadence of AAVSO data is not as high as that of ZTF on these two nights. On December 22, we also observed a transient event with a flipped S-shape profile. Due to the insufficient $(\sim 2 \mathrm{hr})$ time span of the ZTF light curves on these variabilities, we do not perform periodicity analysis for these two nights.

To verify the short-timescale variability of the HO Pup light curve, we performed high-cadence monitoring of HO Pup with the SLT telescope in late 2019-2020. These high-cadence observations were done in a single $r$-band filter with $30 \mathrm{~s}$ exposure time for $2-5 \mathrm{hr}$ continuously on available clear nights. Using both PDM and Lomb-Scargle periodogram routines, the 

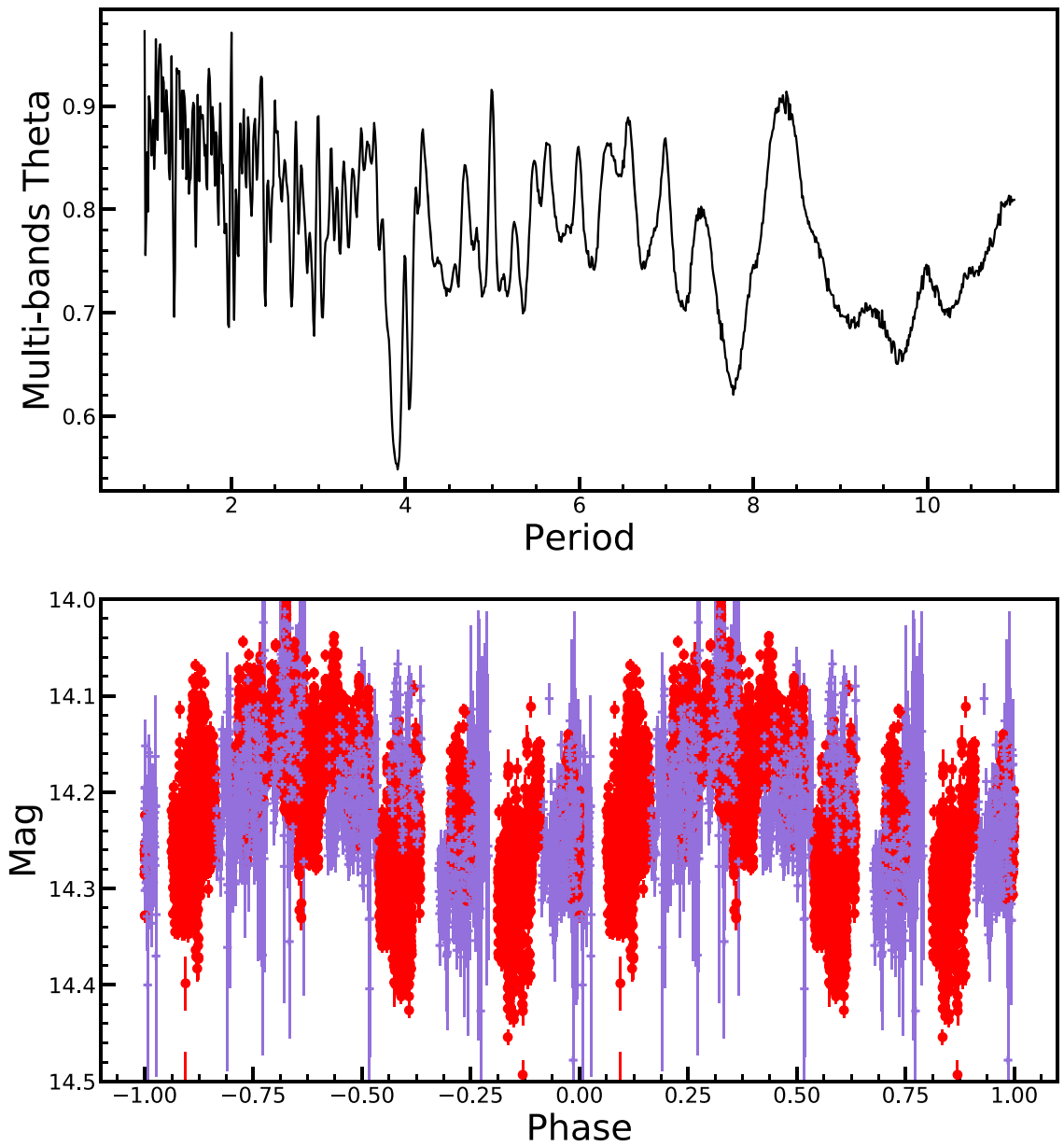

Figure 10. Multiband PDM analysis of the oscillatory variation on a timescale of days. A period of $3.9 \pm 0.005$ days is clearly detected (upper panel) from light curves based on the superposition of two independent observations. The bottom panel is the phased light curves folded with the detected 3.9 day period, in which the symbols are the same as in Figure 2, revealing a sinusoidally shaped light curve.

resolved periodicities are 3.9 days and 50 minutes, respectively, as seen in Figures 10 and 11. Data taken around the brightening (for five nights) and about an hour before the dawn were removed in order to maintain high-quality light curves to detect the short-timescale variability. In addition to the 3.9 day and 50 minute variabilities, two very weak periods were also detected, at 20 minutes and 90 minutes, but cannot be confirmed.

\subsection{Polarization Variation}

We have also observed variations in polarization for HO Pup. Based on polarization level $P$ and position angle $\theta$ measured from Lulin Observatory (see Table 3), a small and yet significant polarization was observed on 2018 October 24 and 26. However, the polarization drops back to an insignificant value on 2018 October 25 and 2018 October 28. The observed variation in polarization suggested that HO Pup exhibits intrinsic polarization.

\section{Discussion}

\subsection{Spectral-energy-distribution Fitting}

Prior to our work, classification of HO Pup in the literature was done via inspection of its light curves, either as a Be star with GCAS-type variability (Samus et al. 2017) or as an IW And-type DN (Kimura et al. 2020a). Here, we first estimated its spectral and luminous class using spectral-energy-distribution fitting together with distance estimation based on the parallax measurement from Gaia Data Release 2 (DR2) $(1.6165 \pm 0.0311 \mathrm{mas}$, or $d=618.6 \mathrm{pc}$, Gaia Collaboration et al. 2018). The observed SED of HO Pup was constructed using available broadband photometry covering from near-UV (NUV) to mid-infrared. In Table 5 we list all available broadband photometric data on HO Pup queried within $1^{\prime \prime}$ radius using the VizieR photometry viewer (Ochsenbein et al. 2000). As shown in Figure 12, the SED peaked between the NUV and $B$ bands, which indicates that it is either a hot earlytype star or a source with a hot component.

Assuming that HO Pup is a luminous B-type main-sequence star, fitting these broadband photometric data with a theoretical SED model (Kurucz 1993) suggested that the spectral class of HO Pup has to be B1V, as demonstrated in Figure 12(a), with the corresponding extinction of $A_{V}=0.5$ and at a distance of $16 \mathrm{kpc}$. However, the distance of $16 \mathrm{kpc}$ is inconsistent with the Gaia DR2 distance. If the Gaia DR2 distance is correct, we then derived $M_{V} \sim 4.7 \mathrm{mag}$ for HO Pup by adopting an apparent magnitude of $m_{V}=13.7 \mathrm{mag}$ and an extinction of $A_{V}=0.1$ mag. This value of absolute magnitude $M_{V}$ is consistent with the known IW And-type stars, as summarized in Table 6. Therefore, the possibility of HOPup being a Be star is ruled out. 


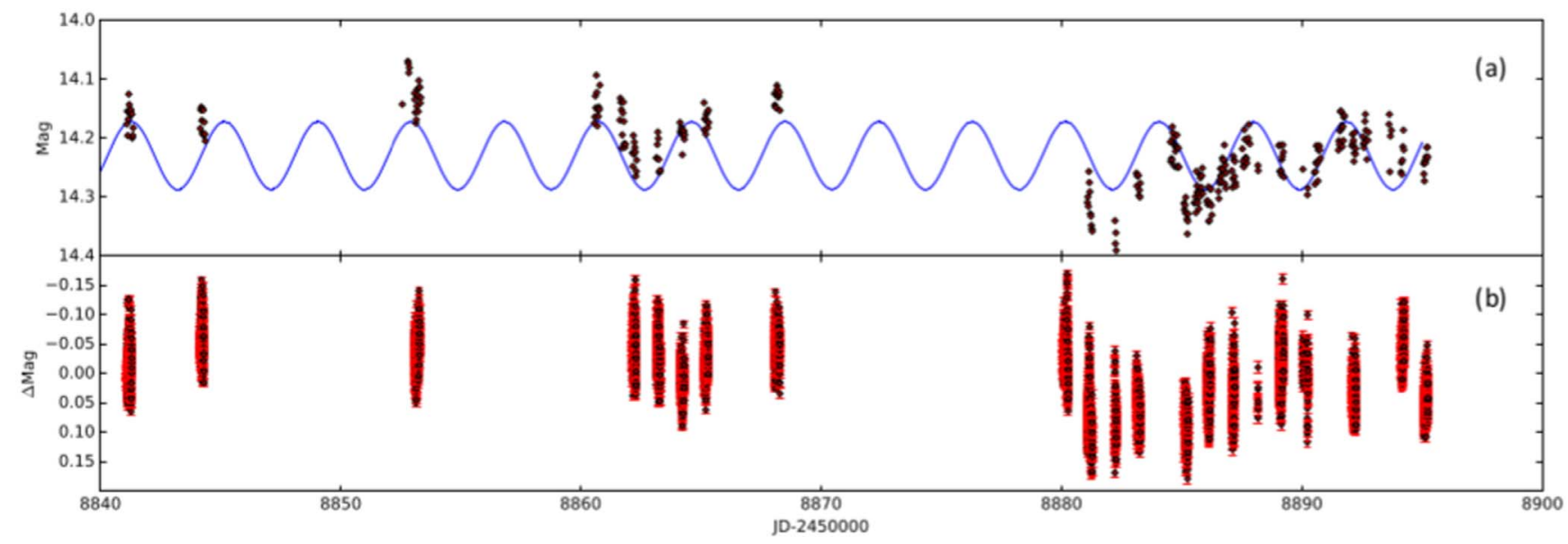

(c)
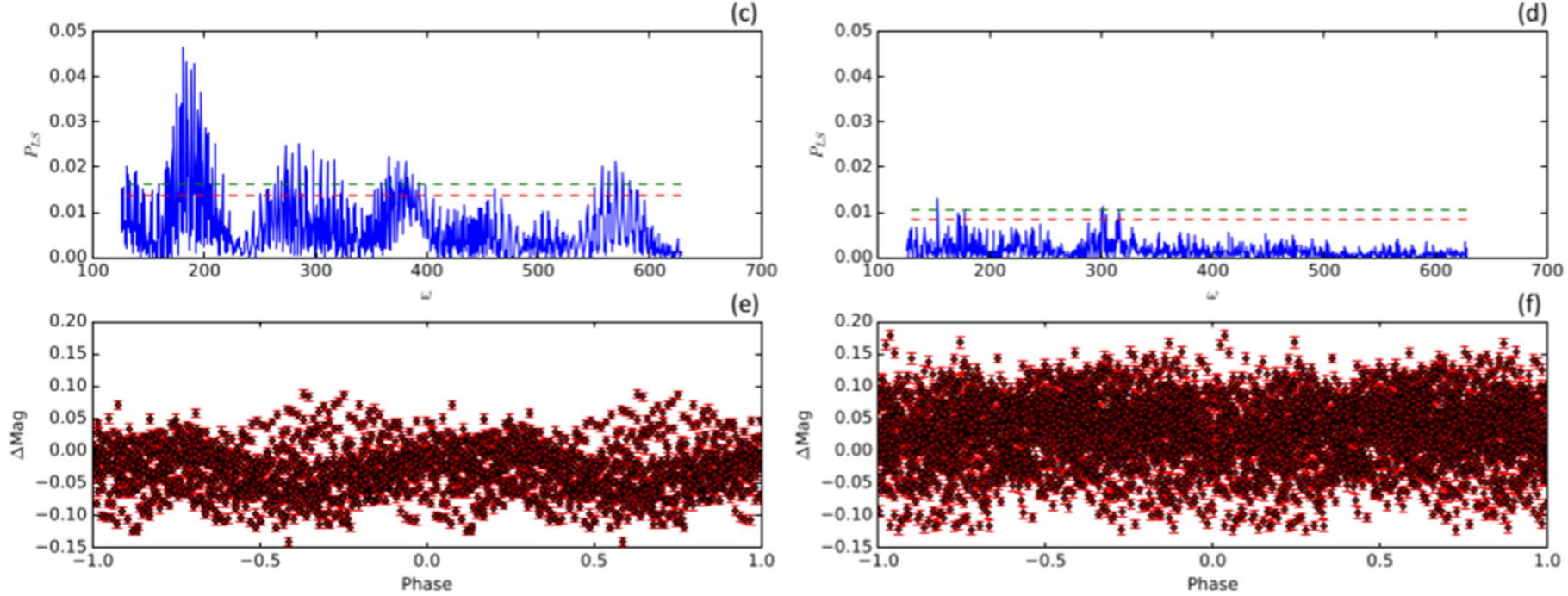

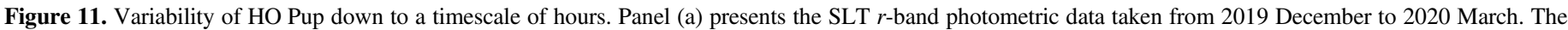

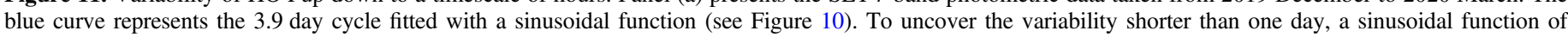

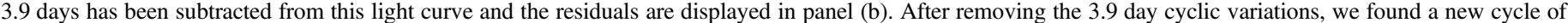

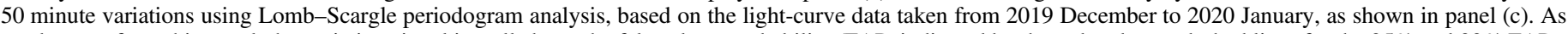

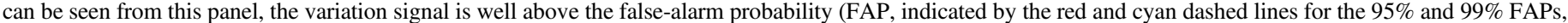

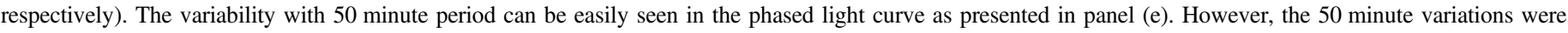

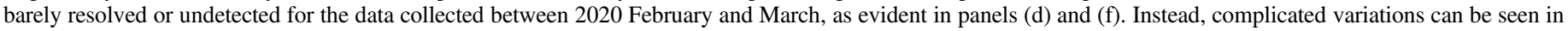
the nightly chunks of light-curve data during this period of observations.

As presented in previous sections, the IW And-type phenomenon can be seen in the light curves of HO Pup collected in our work (as well as in Kimura et al. 2020a). IW And-type DNe, in general, consist of a white dwarf as the primary star, a (low-mass) secondary companion star, and a (hot) disk. In Figure 12(b), a representative theoretical spectrum is shown for a white dwarf at a distance provided by Gaia DR2, $618 \mathrm{pc}$, which has negligible contribution to the observed SED of HO Pup (the black points in Figure 12). Assuming that HO Pup has a hot disk with a temperature of $11,000 \mathrm{~K}$ and a radius of 0.5 solar radius, the blackbody spectrum of such a hot disk can fit well to the observed SED of HO Pup at a distance of 618 pc, as presented in Figure 12(b). We believe this hot disk represents the ensemble average of the disk during the quasi-standstill phase.
The majority of the broadband photometric data listed in Table 5 did not have observation times available. Therefore, photometry at phases of both brightenings and quasi-standstills could possibly be included as part of the observed SED data. ${ }^{15}$ Given that the observed flux of the SED peaked between the NUV band and the $B$ band, it turns out that the observing time in the NUV band is the key to determining which phase we fit in the observed SED, and it was found to be taken on 2012 February 2 (JD =2,455,959.9603; according to the header information available from the corresponding GALEX image). Based on the two data points taken from ASAS-SN with $V \sim 13.9$ mag within 10 days $^{16}$ before and after the NUV

\footnotetext{
$\overline{15}$ For example, the $B$-band data ranged from 13.24 to 15.16 mag (see Table 5), suggesting that one of the brightest data points might be taken during the brightening phase.

16 The next brightening event occurred on 2012 February 17, 14 days after the NUV data were taken. This gap between observation and brightening was shorter than the typical duration of two consecutive HO Pup brightening events, which is longer than 20 days.
} 
Table 5

Collected Broadband Photometric Data for HO Pup Used in SED Fitting

\begin{tabular}{|c|c|c|c|c|}
\hline Band & $\lambda(\mu \mathrm{m})$ & Mag. & Error & Reference \\
\hline NUV & 0.231 & 15.135 & 0.011 & Morrissey et al. (2007) \\
\hline$B j$ & 0.435 & 15.16 & 0.42 & Lasker et al. (2008) \\
\hline$B$ & 0.444 & 14.233 & 0.57 & Zacharias et al. (2012) \\
\hline$B$ & 0.444 & 13.8 & 0.43 & Lasker et al. (2008) \\
\hline$B$ & 0.444 & 13.240 & $\cdots$ & Zacharias et al. (2005) \\
\hline$B f$ & 0.468 & 13.86 & 0.44 & Lasker et al. (2008) \\
\hline$g$ & 0.481 & 14.079 & 0.556 & Geier et al. (2019) \\
\hline$g$ & 0.481 & 14.237 & 0.192 & Heinze et al. (2018) \\
\hline$g$ & 0.481 & 13.818 & 0.273 & Wolf et al. (2018) \\
\hline$g$ & 0.481 & 14.199 & 0.204 & Chambers et al. (2016) \\
\hline$G_{B P}$ & 0.532 & 14.121 & 0.029 & Gaia Collaboration et al. (2018) \\
\hline$V$ & 0.551 & 14.082 & 0.49 & Zacharias et al. (2012) \\
\hline$V$ & 0.551 & 13.340 & $\cdots$ & Zacharias et al. (2005) \\
\hline$r$ & 0.617 & 14.085 & 0.013 & Heinze et al. (2018) \\
\hline$r$ & 0.617 & 13.974 & 0.272 & Wolf et al. (2018) \\
\hline$r$ & 0.617 & 14.043 & 0.008 & Chambers et al. (2016) \\
\hline$r$ & 0.617 & 14.074 & 0.42 & Zacharias et al. (2012) \\
\hline$R$ & 0.658 & 14.170 & $\cdots$ & Zacharias et al. (2005) \\
\hline$G$ & 0.673 & 14.083 & 0.009 & Gaia Collaboration et al. (2018) \\
\hline$i$ & 0.752 & 14.391 & 0.003 & Wolf et al. (2018) \\
\hline$i$ & 0.752 & 14.150 & $\cdots$ & Heinze et al. (2018) \\
\hline$i$ & 0.752 & 14.118 & $\cdots$ & Chambers et al. (2016) \\
\hline$i$ & 0.752 & 13.887 & 0.10 & Zacharias et al. (2012) \\
\hline In & 0.784 & 14.04 & 0.43 & Lasker et al. (2008) \\
\hline$G_{R P}$ & 0.797 & 13.833 & 0.049 & Gaia Collaboration et al. (2018) \\
\hline$z$ & 0.866 & 14.586 & 0.003 & Wolf et al. (2018) \\
\hline$z$ & 0.866 & 14.363 & 0.015 & Heinze et al. (2018) \\
\hline$z$ & 0.866 & 14.311 & 0.017 & Chambers et al. (2016) \\
\hline$y$ & 0.962 & 14.354 & 0.054 & Heinze et al. (2018) \\
\hline$y$ & 0.962 & 14.327 & 0.050 & Chambers et al. (2016) \\
\hline$J$ & 1.25 & 13.300 & 0.026 & Skrutskie et al. (2006) \\
\hline$H$ & 1.65 & 13.137 & 0.027 & Skrutskie et al. (2006) \\
\hline$K_{s}$ & 2.15 & 13.152 & 0.037 & Skrutskie et al. (2006) \\
\hline W1 & 3.40 & 13.480 & 0.028 & Cutri et al. (2012) \\
\hline W2 & 4.60 & 13.358 & 0.038 & Cutri et al. (2012) \\
\hline W3 & 12.00 & 12.564 & 0.471 & Cutri et al. (2012) \\
\hline
\end{tabular}

observation in the quasi-standstill phase, it is very likely that the NUV observation was taken while HO Pup was in the quasi-standstill phase.

Alternatively, the observed SED of HO Pup can also be fitted with a hot sub-luminous star as its secondary, as shown in Figure 12(c). We note that an early spectroscopic observation has suggested that the prototype object, IW And, could be an early-type dwarf or subdwarf (Meinunger 1980).

\subsection{Photometric Characteristics}

Two major phenomena of HO Pup, i.e., brightenings and deep dips, were found to be consistent with models proposed by two different teams-Kimura et al. (2020a) and Hameury \& Lasota (2014). The former team explained the usual status of HO Pup (without presenting the deep dips) based on a tilted accretion disk model inspired by the idea proposed by Kato (2019). The latter team reproduced both the sudden brightening and deep dip by variations of the mass-transfer rate.

In the tilted accretion disk model, the accretion flow from the donor star will fall directly into the inner disk as well as outer disk. With a steady supply of mass, the inner part of the accretion disk of IW And-type stars can stay in a hot state in both quasi-standstill and brightening phases. Once the mass threshold has been achieved in the outer disk, brightening could occur from inside to outside of the disk, known as insideout brightening (Court et al. 2020; Kimura et al. 2020a). The brightening event, in fact, shows up in the light curve (for example, see Figure 3 at JD 2,458,565). Due to the instability of the accretion rate, the entire disk can be cooled since there is little mass left in the disk, and hence the brightness can drop dramatically.

The performance of the thermal-viscous instability in the tilted accretion disk proposed by Kimura et al. (2020a) is consistent with the brightening events and the 3.9 day cycle seen in the light curve of HO Pup. Indeed, Kimura et al. (2020a) stated that the tilted disk models with an accretion rate of $10^{17} \mathrm{~g} \mathrm{~s}^{-1}$ were similar to the "heartbeat-type oscillations" of HO Pup. Furthermore, in their highly tilted disk models with accretion rates of $10^{16.75}$ and $10^{17} \mathrm{~g} \mathrm{~s}^{-1}$ (the $\mathrm{C} 1$ and $\mathrm{C} 2$ models, respectively), the sawtooth-like pattern shown in the quasistandstill phase resembles the light curve of HO Pup, displaying the 3.9 day variations (see Figure 10). However, as pointed out by the referee, the 3.9 day variations of the HO Pup light curve could also be caused by the precession of the tilted disk (the super-orbital modulation). Nevertheless, the tilted disk models did not include eclipsing-like deep-dip events ( $>1 \mathrm{mag}$ ) just after the brightening phase due to the difficulty of reproducing such a one-time dip event in the model.

To explain a similar phenomenon seen in the light curve of V513 Cas, a kind of mass-transfer-burst model has been framed by Hameury \& Lasota (2014), which reproduces the eclipsinglike deep-dip events. With a sudden change of mass-transfer rate, the deep dip appeared in this model, which is similar to HO Pup with drops in brightness by $\sim 2.5 \mathrm{mag}$. It could be the same mechanism responsible for the observed light curves of either V513 Cas or HO Pup, or both. The event profiles of this model seem to be consistent with observations of an abrupt increase followed by a fall below the average. Given that the brightness of HO Pup dropped by $\sim 2.5 \mathrm{mag}$ during the deepdip events, this implies a significant change in the mass-transfer rate, as the luminosity dropped by 10 times during these events. However, we did not find any evidence that the possible mechanisms of mass-transfer bursts, which are listed by Hameury \& Lasota (2014), work in HO Pup. Also, because of the change in the mass-transfer rate, it is expected that the moving average of optical luminosity over a long timescale would be dramatically altered, but no such trend was seen in the HO Pup data (even with a very long baseline). Perhaps long-term high-cadence observations could help in resolving the physical reasons behind these deep-dip events (such as detecting the negative superhumps), especially when such observations are carried out during those stochastic events.

Finally, we pointed out that the brightening cycle of HO Pup varied from 23 to 61 days, which is not shown in either the model from Kimura et al. (2020a) or that from Hameury \& Lasota (2014). If the disk is tilted, the cycle of brightening may change because the geometry of the disk, the tilt angle, or the thickness of the disk could change on a long timescale. Furthermore, while the deep-dip events were observed in the 2017-2018 season, the cycle of brightening before each deepdip event also has a very large variation, lasting from 10 days to 30 days. A good scenario for explaining such variability with cyclic duration of brightening is yet to be constructed. 


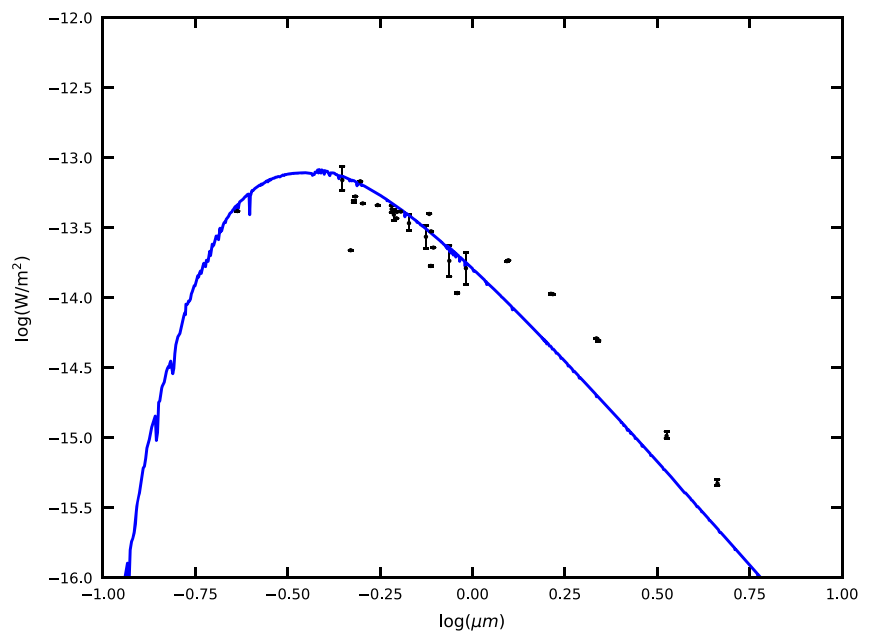

(a) Fitting with a Be star spectrum at $16 \mathrm{kpc}$

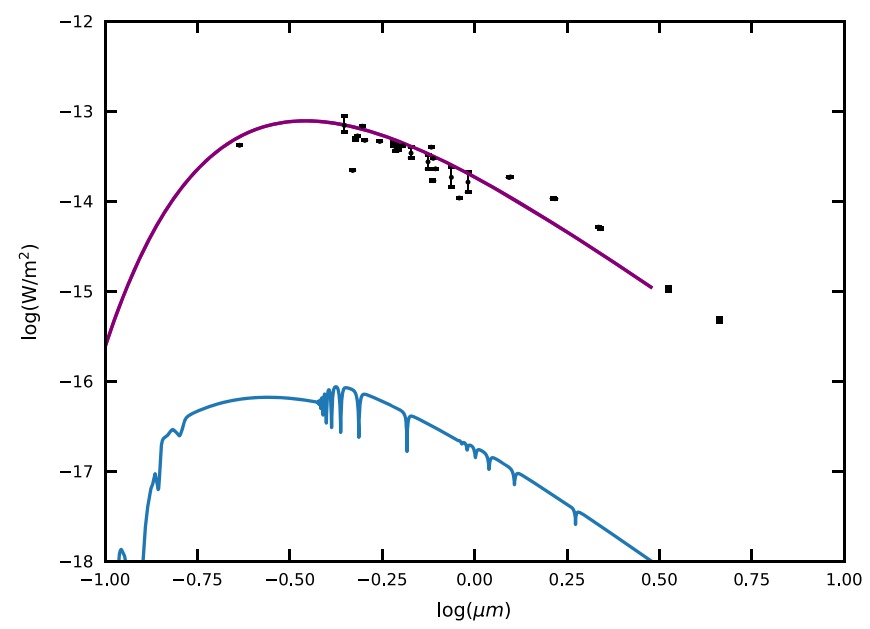

(b) Fitting with a hot disk black-body at $618 \mathrm{pc}$

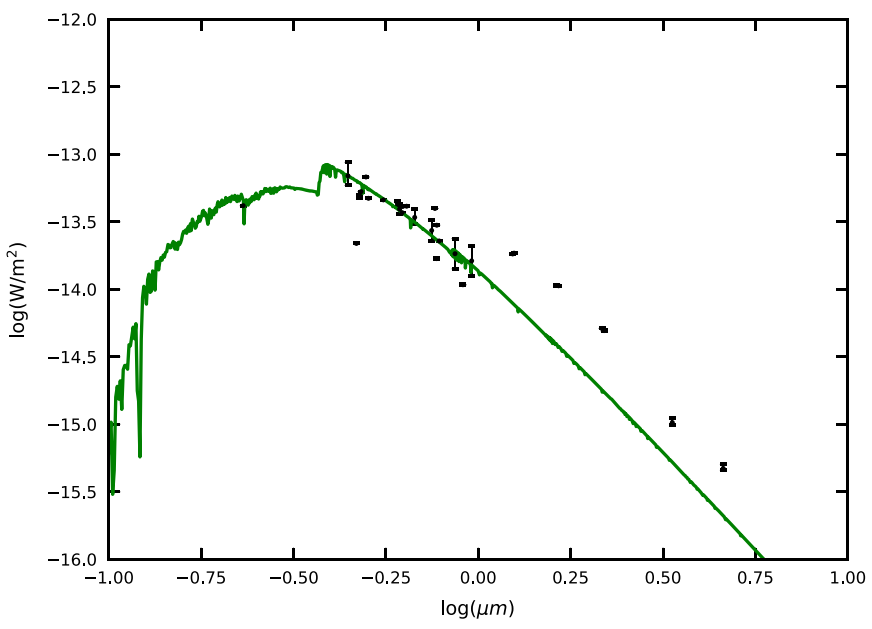

(c) Fitting with a hot sub-luminous star spectrum at $618 \mathrm{pc}$

Figure 12. Fitting of the observed SED of HO Pup (black points from broadband photometry; see Table 5) with various theoretical spectra. In panel (a), the blue curve is the theoretical spectrum for a B1V dwarf, taken from the stellar atmosphere model of Kurucz (1993), at a distance of $16 \mathrm{kpc}$ with $A_{V}=0.5$. In panel (b), the light blue curve is a representative theoretical white dwarf spectrum (with effective temperature of 12,000 K) adopted from Tremblay \& Bergeron (2009) and Koester (2010), available at the Spanish Virtual Observatory, at a distance of $618 \mathrm{pc}$ with $A_{V}=0.1$. The purple curve represents the sum of the white dwarf spectrum and a hot disk with radius of 0.5 solar radius assuming blackbody radiation at $11,000 \mathrm{~K}$, both at the same distance of $618 \mathrm{pc}$ with $A_{V}=0.1$. In panel (c), the green curve is a theoretical spectrum for a hot sub-luminous star with an effective temperature of 12,000 K, taken from the stellar atmosphere model of Kurucz (1993), assuming a distance of $618 \mathrm{kpc}$ with $A_{V}=0.1$.

\subsection{Spectroscopic Characteristics}

As shown in lower panel of Figure 4, the spectra of HO Pup displayed broad Balmer absorption lines up to $\mathrm{H} \zeta$, as well as other helium and metal lines including $\mathrm{He}$ I, He II, Fe I, and $\mathrm{Mg}$ I. Moreover, we found CV signatures with weak emission cores presented in most of the broad Balmer absorption lines from our P200/DBSP spectrum, which was taken at the brightening right after the maximum brightness. Szkody et al. (2013) observed two CVs, IW And and V513 Cas, in their brightening phase, in which their Balmer lines were nearly the same as in HO Pup. Apart from the hydrogen features, there is a double-peaked, bumpy, and shoulder-like feature in between $\mathrm{H} \beta$ and $\mathrm{H} \gamma$, which include the lines of C III, N III (known as Bowen fluorescence at $4640 \AA$ ), and He II ( $4731 \AA$ ). This is a unique blended emission line that can be revealed especially during the phase of $\mathrm{CV}$ brightening. In the phase of quasi- standstill, the Bowen features become much more insignificant as seen in the $\mathrm{BOAO} / \mathrm{BOES}$ spectrum, along with shallower Balmer absorption. Hessman et al. (1984) reported timeresolved spectroscopic observations from brightening to quasistandstill for a classical CV, SS Cyg, showing the strength of the emissions from Bowen fluorescence declining to the continuum level. The similarity of HO Pup spectral features and these CV features strongly suggested that HOPup is indeed an IW And-type star. Two additional spectroscopic differences between brightenings and quasi-standstill phase not seen in the literature are the weaker H I $6678 \AA$ line shown in the quasi-standstill phase and the $\mathrm{Na}$ I doublet appearing during the brightenings (see Figure 4). 
Table 6

Absolute Magnitudes of IW And-type Stars

\begin{tabular}{lcccc}
\hline \hline Name & Distance $(\mathrm{pc})$ & $A_{V}$ & $m_{V}$ & $M_{V}$ \\
\hline IW And & 835.1 & $0.152^{\mathrm{a}}$ & $14.565^{\mathrm{b}}$ & 4.804 \\
HO Pup & 618.6 & $0.091^{\mathrm{a}}$ & $13.740^{\mathrm{c}}$ & 4.692 \\
IM Eri & 191.2 & $0.000^{\mathrm{a}}$ & $11.771^{\mathrm{c}}$ & 5.364 \\
FY Vul & 603.8 & $1.095^{\mathrm{a}}$ & $13.750^{\mathrm{d}}$ & 3.751 \\
V507 Cyg & 613.2 & $0.578^{\mathrm{a}}$ & $14.753^{\mathrm{b}}$ & 5.237 \\
ST Cha & 715.4 & $0.460^{\mathrm{c}}$ & $14.250^{\mathrm{c}}$ & 4.517 \\
V513 Cas & 845.0 & $2.159^{\mathrm{a}}$ & $15.870^{\mathrm{b}}$ & 4.077 \\
KIC 9406652 & 351.5 & $0.030^{\mathrm{a}}$ & $11.710^{\mathrm{c}}$ & 3.905 \\
\hline
\end{tabular}

Notes. Distances were converted from Gaia DR2 parallaxes (Gaia Collaboration et al. 2018).

${ }^{\mathrm{a}}$ Green et al. (2019).

${ }^{\mathrm{b}}$ Henden et al. (2015).

c Jayasinghe et al. (2018).

d Alfonso-Garzón et al. (2012).

Table 7

The Spectroscopic and Photometric Features Shown in Be Stars, (confirmed) IW And-type Stars (Excluding HO Pup), and HO Pup

\begin{tabular}{lccc}
\hline \hline & Be Stars & IW And Stars & HO Pup \\
\hline Number & $\sim 3000$ & $7+7^{\mathrm{a}}$ & 1 \\
Hydrogen lines & yes & yes & yes \\
Helium lines & yes & yes & yes \\
Spectral type & early & early & early \\
Bowen fluorescence & no & yes & yes \\
Dip $\delta M_{V}$ & 4 mag & 2.5 mag & 2.5 mag \\
& (RW Tau $)$ & $15-55$ days & $7-30$ days \\
Dip cycle & $\ldots$ & $($ ST Cha $)$ & \\
& & $0.5-1$ mag & 0.5 mag \\
Brightening $\delta M_{V}$ & $>0.5$ mag & $15-100$ days & $23-61$ days \\
Brightening cycle & 71 days $(\delta$ Sco) & a few days & $\sim 5$ days \\
Brightening duration & $2-1000$ days & & \\
\hline
\end{tabular}

Note.

a The first "7" represents the confirmed IW And-type stars as mentioned in the Introduction; the second " 7 " is counting the candidates listed in footnote 11.

\subsection{Polarimetric Characteristics}

Based on the polarimetric observations taken during the quasi-standstill phase (see upper panel of Figure 4), HO Pup exhibited an intrinsic optical polarization variability, in which the polarized light included the light from both the star and the disk. The presence of a magnetic field or disk scattering may account for the observed polarization. Despite the variation of polarization level, the polarization percentages $P$ on four different nights were all measured to be less than $1 \%$ in our $r$ and $i$-band TRIPOL2 data. This level of polarization has been observed in three other DNe during their quasi-standstill phase (Szkody et al. 1982).

\subsection{Similarity to Be Stars}

Previously, HO Pup was considered as a Be star based on its GCAS-like light curves. However, those incomplete light curves were sparsely sampled in the past, as shown in Figure 2, and this could be responsible for the misclassification of HO Pup as a Be star. HO Pup has better observation coverage since 2016, and therefore its DN nature is revealed by Kimura et al. (2020a) from the dense sampling of the light curve.
Since both Be stars and IW And-type stars are hot objects with hydrogen and helium lines, and exhibit irregular and abrupt photometric variations (e.g., due to the presence of a disk), these two types of stars share a number of common observational features. As is evident from the case of HO Pup, an IW And-type star could be misclassified as a Be star based on limited observational evidence (and vice versa). In Table 7, we compare and summarize some of the important observational features of Be stars, IW And-type stars, and HO Pup. For example, Be stars could display regular dip events due to eclipsing as in the case of RW Tau with dips fainter by $\sim 4$ mag (Bookmyer 1977). On the other hand, the deepest dip event observed on IW And-type stars is fainter by $\sim 3$ mag (Kato 2019) without cyclic variations. The observed dip events of HO Pup, without any regularity in their cycle (from 12 to 30 days), have a greater similarity to IW And-type stars than to eclipsing Be stars. Furthermore, for the brightening events that were not followed by dip events, IW And-type stars have much more regularity in their brightening phase (with brightening cycles ranging from 15 to 200 days) than Be stars that display a GCAS-type light curve, in which the brightenings are more secular.

Although the brightening durations of IW And-type stars are mostly much shorter than those of Be stars, they have a similar inverse correlation between photometric brightness and spectroscopic line emission. For IW And-type stars, the brightness variation is dominated by an accretion disk along with the growth of the disk atmosphere, so we see the growth of hydrogen absorption. On the other hand, disk extinction dominates the brightness variation for Be stars (Sigut \& Patel 2013). After the disk dissipates, we see brighter Be stars with less surrounding line-emitting material. The inside-out mass processing is the mechanism by which a Be star forms a "decretion" disk surrounding the host star (Rivinius et al. 2013). It is very interesting to see the two very different kinds of disk (accretion disk and decretion disk) showing similar photometric and spectroscopic behavior.

We see a similar interplay during the eight nights of our CFHT/ESPaDOnS observations. During these observations, HO Pup experienced a transition from 14 to $13.5 \mathrm{mag}$ at the same time as the $\mathrm{EW}$ of $\mathrm{H} \alpha$ line strength decreased from $-6.4 \AA$ to $-1.2 \AA$ (see Figure 3 ). A similar behavior between $\mathrm{H} \alpha$ emission line strength and photometric brightness was also clearly observed in another Be star, V438 Aur (Labadie-Bartz et al. 2017). However, the obscured phase of HO Pup lasts up to $\sim 36$ days, which is far less than that of V438 Aur (more than 2000 days).

Therefore, as summarized in Table 7, we cannot easily distinguish IW And-type stars from the field (luminous) Be stars without knowing their distance, because they share the same color and nearly the same spectral features. With the low sampling of light curves and limited spectroscopic observations, neither photometric nor spectroscopic data can be used to distinguish possible IW And-type stars from a list of faint Be stars. If we observe any other unknown IW And-type stars with a low-sampling light curve (as in the case of HO Pup before 2010), there is a large probability that these stars would be classified as Be stars based on their GCAS-like light curve. It might also be difficult to separate these two classes of stars with spectroscopic observations, unless the spectra were taken during the brightening phase and contain unique features of metal lines (such as Bowen fluorescence) for CVs. Since the 
brightening phases of a CV last only a few days, whereas they can last for longer than 10 days in Be stars, there is a narrow observing window in which to take the spectra of these objects.

\section{Summary}

Instead of classifying $\mathrm{HO}$ Pup as a Be candidate, we confirmed that it is an IW And-type star based on the lightcurve pattern and spectroscopic characterizations, along with the Gaia DR2 distance and SED fitting. As in other IW Andtype stars, light curves of HO Pup display various types of stochastic and quasi-periodic variations such as brightenings, dip events, and a quasi-standstill phase. To shed light on the stellar physics behind the characteristic light curve of IW Andtype stars, further well-covered spectroscopic monitoring along with intense photometric observations are highly desirable.

This work is partly supported by the Ministry of Science and Technology (Taiwan) under grants of 104-2923-M-008-004MY5, 107-2119-M-008-014-MY2, 107-2119-M-008-012, 1082811-M-008-546, and 109-2112-M-155-001. We thank Abert Kong for pointing out the DASCH light curve. We are also grateful for the discussions with Yi Chou, Wen-Ping Chen, Shih-Yun Tang, Michihiro Takami, Chi-Hung Yan, Paula Szkody, and Melissa Graham on this work, as well as suggestions from an anonymous referee to improve the manuscript.

Based on observations obtained with the Samuel Oschin Telescope 48 inch and the 60 inch Telescope at the Palomar Observatory as part of the Zwicky Transient Facility project. ZTF is supported by the National Science Foundation under grant No. AST-1440341 and a collaboration including Caltech, IPAC, the Weizmann Institute for Science, the Oskar Klein Center at Stockholm University, the University of Maryland, the University of Washington, Deutsches Elektronen-Synchrotron and Humboldt University, Los Alamos National Laboratories, the TANGO Consortium of Taiwan, the University of Wisconsin at Milwaukee, and Lawrence Berkeley National Laboratories. Operations are conducted by COO, IPAC, and UW. SED Machine is based upon work supported by the National Science Foundation under grant No. 1106171

This work was partly supported by the GROWTH (Global Relay of Observatories Watching Transients Happen) project funded by the National Science Foundation under PIRE grant No. 1545949. GROWTH is a collaborative project among California Institute of Technology (USA), University of Maryland College Park (USA), University of Wisconsin Milwaukee (USA), Texas Tech University (USA), San Diego State University (USA), University of Washington (USA), Los Alamos National Laboratory (USA), Tokyo Institute of Technology (Japan), National Central University (Taiwan), Indian Institute of Astrophysics (India), Indian Institute of Technology Bombay (India), Weizmann Institute of Science (Israel), The Oskar Klein Centre at Stockholm University (Sweden), Humboldt University (Germany), Liverpool John Moores University (UK), and University of Sydney (Australia).

This research relied on the SIMBAD and VizieR catalog access tool and the Aladin plot tool at CDS, Strasbourg (France), and NASA ADS bibliographic services. This publication makes use of data products from the Wide-field Infrared Survey Explorer, which is a joint project of the University of California, Los Angeles, and the Jet Propulsion Laboratory/California Institute of Technology, funded by the
National Aeronautics and Space Administration. We also made use of data collected at Lulin Observatory, partly supported by MoST grant 108-2112-M-008-001. We sincerely thank the staff and queue observers (Chi-Sheng Lin, Hsiang-Yao Hsaio, and Wei-Jie Hou) at the Lulin Observatory for carrying out the observations with the SLT telescope. We thank the observer at the P200 Telescope, Marianne Heida, for taking the P200/ DBSP spectrum. We also thank staffs of BOAO. We appreciate the achievements and online catalogs provided by ASAS-SN, AAVSO, and Pan-STARRS, making this investigation possible for much longer baseline and/or better time coverage. The DASCH project at Harvard is grateful for partial support from NSF grants AST-0407380, AST-0909073, and AST-1313370.

\section{ORCID iDs}

Chien-De Lee (iD https://orcid.org/0000-0002-3142-7299

Po-Chieh Yu (ib https://orcid.org/0000-0001-8894-0854 Chow-Choong Ngeow (iD https://orcid.org/0000-00018771-7554

Hyun-il Sung (iD https://orcid.org/0000-0001-9515-3584

Jan van Roestel (i) https://orcid.org/0000-0002-2626-2872

Matthew J. Graham (i) https://orcid.org/0000-0002-3168-0139

Dmitry A. Duev (iD https://orcid.org/0000-0001-5060-8733

Thomas Kupfer (i) https://orcid.org/0000-0002-6540-1484

Russ R. Laher (iD https://orcid.org/0000-0003-2451-5482

Frank J. Masci (1) https://orcid.org/0000-0002-8532-9395 Przemek Mróz (1) https://orcid.org/0000-0001-7016-1692 James D. Neill (iD https://orcid.org/0000-0002-0466-1119 Reed Riddle (iD https://orcid.org/0000-0002-0387-370X Ben Rusholme (i) https://orcid.org/0000-0001-7648-4142 Richard Walters (iD https://orcid.org/0000-0002-1835-6078

\section{References}

Alfonso-Garzón, J., Domingo, A., Mas-Hesse, J. M., et al. 2012, A\&A, 548, A79

Bellm, E. C., Kulkarni, S. R., Graham, M. J., et al. 2019, PASP, 131, 018002 Bellm, E. C., \& Sesar, B. 2016, Pyraf-Dbsp: Reduction Pipeline for the Palomar Double Beam Spectrograph, Astrophysics Source Code Library, ascl:1602.002

Ben-Ami, S., Konidaris, N., Quimby, R., et al. 2012, Proc. SPIE, 844686 Blagorodnova, N., Neill, J. D., Walters, R., et al. 2018, PASP, 130, 035003 Bookmyer, B. B. 1977, PASP, 89, 533

Cenko, S. B., Fox, D. B., Moon, D.-S., et al. 2006, PASP, 118, 1396

Chambers, K. C., Magnier, E. A., Metcalfe, N., et al. 2016, arXiv:1612.05560 Court, J. M. C., Scaringi, S., Littlefield, C., et al. 2020, MNRAS, 494, 4656 Cutri, R. M., et al. 2012, yCat, 2311, 0

Donati, J.-F., Jardine, M. M., Gregory, S. G., et al. 2007, MNRAS, 380, 1297 Donati, J.-F., Semel, M., Carter, B. D., Rees, D. E., \& Collier Cameron, A. 1997, MNRAS, 291, 658

Gaia Collaboration, Brown, A. G. A., Vallenari, A., et al. 2018, A\&A, 616, A1 Geier, S., Raddi, R., Gentile Fusillo, N. P., et al. 2019, A\&A, 621, A38 Gies, D. R., Guo, Z., Howell, S. B., et al. 2013, ApJ, 775, 64

Graham, M. J., Kulkarni, S. R., Bellm, E. C., et al. 2019, PASP, 131, 078001 Green, G. M., Schlafly, E., Zucker, C., et al. 2019, ApJ, 887, 93

Grindlay, J., Tang, S., Los, E., et al. 2012, IAU Symp. 285, New Horizons in Time-Domain Astronomy (Cambridge: Cambridge Univ. Press), 29 Hambsch, F.-J. 2012, JAVSO, 40, 1003

Hameury, J.-M., \& Lasota, J.-P. 2014, A\&A, 569, A48

Heinze, A. N., Tonry, J. L., Denneau, L., et al. 2018, AJ, 156, 241

Henden, A. A., Levine, S., Terrell, D., et al. 2015, AAS Meeting, 225, 336.16 Hessman, F. V., Robinson, E. L., Nather, R. E., et al. 1984, ApJ, 286, 747

Huang, P. 2019, PhD thesis, Graduate Institute of Astronomy, National Central Univ., Taiwan

Jayasinghe, T., Kochanek, C. S., Stanek, K. Z., et al. 2018, MNRAS, 477, 3145 Kaiser, N., Burgett, W., Chambers, K., et al. 2010, Proc. SPIE, 77330E Kato, T. 2019, PASJ, 71, 20

Kim, K.-M., Jang, B.-H., Han, I., et al. 2002, JKAS, 35, 221 
Kimura, M., Osaki, Y., Kato, T., et al. 2020a, PASJ, 72, 22

Kimura, M., Osaki, Y., \& Kato, T. 2020b, PASJ, 72, 94

Kochanek, C. S., Shappee, B. J., Stanek, K. Z., et al. 2017, PASP, 129, 104502

Koester, D. 2010, MmSAI, 81, 921

Kukarkin, B. V., Kholopov, P. N., Pskovsky, Y. P., et al. 1971, General Catalogue of Variable Stars, Vol. III (3rd ed.; Moskva: Astronomical Council of the Academy of Sciences in the USSR)

Kulkarni, S. R. 2013, ATel, 4807, 1

Kurucz, R. 1993, ATLAS9 Stellar Atmosphere Programs and $2 \mathrm{~km} / \mathrm{s}$ grid Kurucz CD-ROM No. 13 (Cambridge, MA: Smithsonian Astrophysical Observatory), 13

Labadie-Bartz, J., Pepper, J., McSwain, M. V., et al. 2017, AJ, 153, 252

Lasker, B. M., Lattanzi, M. G., McLean, B. J., et al. 2008, AJ, 136, 735

Law, N. M., Kulkarni, S. R., Dekany, R. G., et al. 2009, PASP, 121, 1395

Mainzer, A., Bauer, J., Grav, T., et al. 2011, ApJ, 731, 53

Manek, J. 1997, IBVS, 4476, 1

Masci, F. J., Laher, R. R., Rusholme, B., et al. 2019, PASP, 131, 018003

Mason, E., \& Howell, S. B. 2016, A\&A, 589, A106

Meinunger, L. 1980, IBVS, 1795, 1

Morrissey, P., Conrow, T., Barlow, T. A., et al. 2007, ApJS, 173, 682

Ngeow, C.-C., Lee, C.-D., Yu, P.-C., et al. 2019, JPhCS, 1231, 012010

Ochsenbein, F., Bauer, P., \& Marcout, J. 2000, A\&AS, 143, 23

Oke, J. B., \& Gunn, J. E. 1982, PASP, 94, 586

Pojmanski, G. 1997, AcA, 47, 467

Pojmanski, G. 2002, AcA, 52, 397
Pojmanski, G., Pilecki, B., \& Szczygiel, D. 2005, AcA, 55, 275

Rigault, M., Neill, J. D., Blagorodnova, N., et al. 2019, A\&A, 627, A115

Ritter, A., Ngeow, C. C., Konidaris, N., et al. 2014, CoSka, 43, 209

Rivinius, T., Carciofi, A. C., \& Martayan, C. 2013, A\&ARv, 21, 69

Samus, N. N., Kazarovets, E. V., Durlevich, O. V., Kireeva, N. N., \& Pastukhova, E. N. 2017, ARep, 61, 80

Sato, S., Chieh Huang, P., Chen, W. P., et al. 2019, RAA, 19, 136

Schlegel, E. M., \& Honeycutt, R. K. 2019, ApJ, 876, 152

Schmidt, G. D., Elston, R., \& Lupie, O. L. 1992, AJ, 104, 1563

Shappee, B. J., Prieto, J. L., Grupe, D., et al. 2014, ApJ, 788, 48

Sigut, T. A. A., \& Patel, P. 2013, ApJ, 765, 41

Skrutskie, M. F., Cutri, R. M., Stiening, R., et al. 2006, AJ, 131, 1163

Stellingwerf, R. F. 1978, ApJ, 224, 953

STScI Development Team 2013, Pysynphot: Synthetic Photometry Software Package, Astrophysics Source Code Library, ascl:1303.023

Szkody, P., Albright, M., Linnell, A. P., et al. 2013, PASP, 125, 1421

Szkody, P., Michalsky, J. J., \& Stokes, G. M. 1982, PASP, 94, 137

Tremblay, P.-E., \& Bergeron, P. 2009, ApJ, 696, 1755

Wolf, C., Onken, C. A., Luvaul, L. C., et al. 2018, PASA, 35, e010

Yu, P. C., Lin, C. C., Chen, W. P., et al. 2015, AJ, 149, 43

Yu, P.-C., Lin, C.-C., Lin, H.-W., et al. 2016, AJ, 151, 121

Yu, P.-C., Yu, C.-H., Lee, C.-D., et al. 2018, AJ, 155, 91

Zacharias, N., Finch, C. T., Girard, T. M., et al. 2012, yCat, 1322, 0

Zacharias, N., Monet, D. G., Levine, S. E., et al. 2005, yCat, 1297, 